\title{
HECKE CORRESPONDENCE, STABLE MAPS AND THE KIRWAN DESINGULARIZATION
}

\author{
YOUNG-HOON KIEM
}

\begin{abstract}
We prove that the moduli space $\overline{\mathbf{M}}_{0,0}(\mathcal{N}, 2)$ of stable maps of degree 2 to the moduli space $\mathcal{N}$ of rank 2 stable bundles of fixed determinant over a smooth projective curve of genus $g$ has two irreducible components which intersect transversely. One of them is Kirwan's partial desingularization $\widetilde{\mathcal{M}}_{X}$ of the moduli space $\mathcal{M}_{X}$ of rank 2 semistable bundles with determinant isomorphic to $\mathcal{O}_{X}(y-x)$ for some $y \in X$. The other component is the partial desingularization of $\mathbb{P H o m}\left(\operatorname{sl}(2)^{\vee}, \mathcal{W}\right) / / P G L(2)$ for a vector bundle $\mathcal{W}=R^{1} \pi_{*} \mathcal{L}^{-2}(-x)$ of rank $g$ over the Jacobian of $X$. We also show that the Hilbert scheme $\mathbf{H}$, the Chow scheme $\mathbf{C}$ of conics in $\mathcal{N}$ and $\overline{\mathbf{M}}_{0,0}(\mathcal{N}, 2)$ are related by explicit contractions.
\end{abstract}

\section{INTRODUCTION}

Let $X$ be a smooth projective curve of genus $g \geq 3$ over the complex number field. We fix $x \in X$ throughout this paper. The moduli space $\mathcal{N}$ of rank 2 stable bundles over $X$ with determinant $\mathcal{O}_{X}(-x)$ is a smooth projective Fano variety whose Picard group is generated by a very ample line bundle $\Theta$. (See 2].) The problem that we address in this paper is the following.

Problem 1.1. Describe explicitly the moduli space $\overline{\mathbf{M}}_{0,0}(\mathcal{N}, d)$ of stable maps to $\mathcal{N}$ of genus 0 and degree $d$.

Obviously the degree of a stable map $f: C \rightarrow \mathcal{N}$ is defined as the degree of $f^{*} \Theta$. The expected dimension of $\overline{\mathbf{M}}_{0,0}(\mathcal{N}, d)$ is $3 g-6+2 d$ by Riemann-Roch since $c_{1}(T \mathcal{N})=2 \Theta$. When $d=1$, V. Munoz [12 proved that $\overline{\mathbf{M}}_{0,0}(\mathcal{N}, 1)$ is isomorphic to a Grassmannian bundle $\operatorname{Gr}(2, \mathcal{W})$ over $J=P i c^{0}(X)$. (See also 14.) Here $\mathcal{W}=R^{1} \pi_{J *} \mathcal{L}^{-2}(-x)$ is a vector bundle of rank $g$ over $J$ where $\mathcal{L}$ is a Poincaré bundle over $J \times X$ and $\pi_{J}: J \times X \rightarrow J$ is the projection.

The purpose of this paper is to study the $d=2$ case. This is particularly interesting because of Hecke correspondence which has been one of the most powerful tools in the study of moduli spaces of bundles. Let $\mathcal{M}_{X}$ be the moduli space of rank 2 semistable bundles $E$ with $\operatorname{det} E \cong \mathcal{O}_{X}(y-x)$ for some $y \in X$. This is a $(3 g-2)$-dimensional singular projective normal

Date: November 30, 2005.

Partially supported by KRF grant 2005-070-C00005. 
variety which fits into a Cartesian diagram

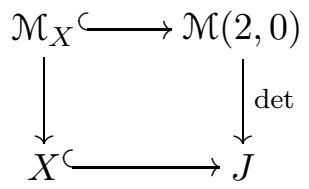

where $M(2,0)$ is the moduli space of semistable bundles of rank 2 and degree 0 over $X$ and the bottom map is $y \mapsto \mathcal{O}_{X}(y-x)$. Hecke correspondence refers to a diagram

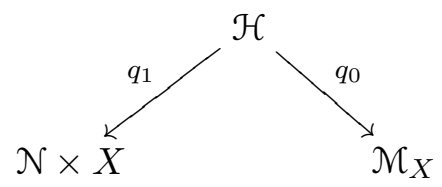

where $q_{1}$ is the projectivization of a universal bundle over $\mathcal{N} \times X$ and $q_{0}$ is a $\mathbb{P}^{1}$-bundle over the stable part $\mathcal{M}_{X}^{s}$. Hence for any $\theta \in \mathcal{M}_{X}^{s}$, we have a stable map

$$
q_{0}^{-1}(\theta) \stackrel{q_{1}}{\longrightarrow} \mathcal{N} \times X \stackrel{\pi_{\mathcal{N}}}{\longrightarrow} \mathcal{N}
$$

which is of degree 2 ([13]). By (1.1), $\mathcal{M}_{X}^{s}$ parameterizes stable maps to $\mathcal{N}$ and hence we have a morphism

$$
\mathcal{M}_{X}^{s} \rightarrow \overline{\mathbf{M}}_{0,0}(\mathcal{N}, 2)
$$

which is injective and birational onto an irreducible component. We prove that this component is precisely Kirwan's partial desingularization $\widetilde{\mathcal{M}}_{X}$ of $\mathcal{M}_{X}$.

Kirwan's partial desingularization is a systematic way to partially resolve the singularities of GIT quotients. The partial desingularization $\tilde{\mathcal{M}}_{X}$ of $\mathcal{M}_{X}$ (see [10]) is the consequence of two blowups first along the deepest stratum

$$
\tilde{X}:=\left\{\xi \in J \mid \xi^{2} \cong \mathcal{O}_{X}(y-x) \text { for some } y \in X\right\}
$$

which is the locus of bundles $\xi \oplus \xi, \xi \in \tilde{X}$ and then along the proper transform of the middle stratum

$$
\mathcal{K}_{J}:=\left\{\left(\xi_{1}, \xi_{2}\right) \in J \times J \mid \xi_{1} \xi_{2} \cong \mathcal{O}_{X}(y-x) \text { for some } y \in X\right\} / \mathbb{Z}_{2}
$$

which is the locus of bundles $\xi_{1} \oplus \xi_{2},\left(\xi_{1}, \xi_{2}\right) \in \mathcal{K}_{J}$ where $\mathbb{Z}_{2}$ interchanges $\xi_{1}$ and $\xi_{2}$. The upshot is an orbifold $\widetilde{\mathcal{M}}_{X}$ which is singular along a nonsingular subvariety $\Delta_{\tilde{X}} \cong \mathbb{P}\left(S^{2} \mathcal{A}_{\tilde{X}}^{\vee}\right)$ where $\mathcal{A}_{\tilde{X}} \rightarrow G r\left(2, \mathcal{W}_{0}\right)$ is the universal rank 2 bundle on the relative Grassmannian of the bundle $\mathcal{W}_{0}=R^{1} \pi_{*}\left(\mathcal{L}_{0}^{-2}(-x)\right)$, $\mathcal{L}_{0}$ being the Poincaré line bundle over $\tilde{X} \times X$ and $\pi: \tilde{X} \times X \rightarrow \tilde{X}$ being the projection. By blowing up $\widetilde{\mathcal{M}}_{X}$ along $\Delta_{\tilde{X}}$ we obtain a (full) desingularization $\widehat{\mathcal{M}}_{X}$ of $\mathcal{M}_{X}$. The exceptional divisor of the last blowup is

$$
\widehat{\Delta}_{\tilde{X}}=\mathbb{P}\left(S^{2} \mathcal{A}_{\tilde{X}}^{\vee}\right) \times_{G r\left(2, \mathcal{W}_{0}\right)} \mathbb{P}\left(\mathcal{W}_{0} / \mathcal{A}_{\tilde{X}} \oplus \eta\right)
$$


for some line bundle $\eta$ over $\operatorname{Gr}\left(2, \mathcal{W}_{0}\right)$ and the normal bundle is $\mathcal{O}(-1,-2)$ on the fibers $\mathbb{P}^{2} \times \mathbb{P}^{g-2}$ over $\operatorname{Gr}\left(2, \mathcal{W}_{0}\right)$. The blowup $\widehat{\mathcal{M}}_{X} \rightarrow \widetilde{\mathcal{M}}_{X}$ is the contraction of $\mathbb{P}\left(\mathcal{W}_{0} / \mathcal{A}_{\tilde{X}} \oplus \eta\right)$.

Although the partial desingularization $\tilde{\mathcal{M}}_{X}$ has been quite useful especially for cohomological computations, its moduli theoretic meaning has been unknown. We prove the following in this paper.

Theorem 1.2. (1) The partial desingularization $\widetilde{\mathcal{M}}_{X}$ is isomorphic to the irreducible component of $\overline{\mathbf{M}}_{0,0}(\mathcal{N}, 2)$ containing the image of $\mathcal{M}_{X}^{s}$.

(2) We have

$$
\overline{\mathbf{M}}_{0,0}(\mathcal{N}, 2) \cong \widetilde{\mathcal{M}}_{X} \cup \widetilde{Q}_{J}
$$

where $\widetilde{Q}_{J}=\overline{\mathbf{M}}_{0,0}(\mathbb{P} \mathcal{W} / J, 2)$ is the partial desingularization of the GIT quotient $Q_{J}=\mathbb{P} \operatorname{Hom}\left(s l(2)^{\vee}, \mathcal{W}\right) / / P G L(2)$. The two irreducible components intersect transversely along the partial desingularization $\widetilde{Q}_{\tilde{X}}=\overline{\mathbf{M}}_{0,0}\left(\mathbb{P} \mathcal{W}_{0} / \tilde{X}, 2\right)$ of the GIT quotient $Q_{\tilde{X}}=\mathbb{P H o m}\left(\operatorname{sl}(2)^{\vee}, \mathcal{W}_{0}\right) / / P G L(2)$.

In [6], a strategy for computing the intersection pairing of the partial desingularization of a GIT quotient is provided and the intersection pairing of the partial desingularization of the moduli space $\mathcal{M}\left(2, \mathcal{O}_{X}\right)$ was studied in [7. By Theorem [1.2, the results of [7] should give us the second order deformation of the quantum cohomology ring of $\mathcal{N}$. Since the quantum cohomology ring is in principle understood [12, we didn't pursue the computation.

A problem closely related to Problem 1.1 is the following.

Problem 1.3. Describe explicitly the Hilbert scheme and the Chow scheme of curves of degree $d$, i.e. subschemes of $\mathcal{N}$ with Hilbert polynomial $d m+1$ with respect to the ample generator $\Theta$.

When $d=1$, they all coincide with $\overline{\mathbf{M}}_{0,0}(\mathcal{N}, 1)$. We prove the following for conics (degree 2 curves).

Theorem 1.4. The Hilbert scheme $\mathbf{H}$ of conics, the Chow scheme $\mathbf{C}$ of conics and $\mathbf{M}:=\overline{\mathbf{M}}_{0,0}(\mathcal{N}, 2)$ are related by contractions

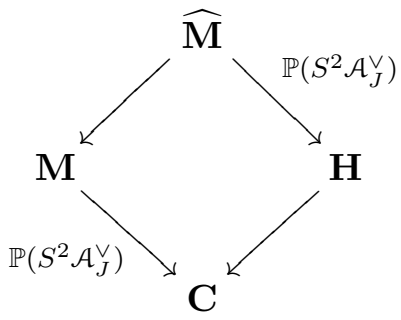

where $\widehat{\mathbf{M}}$ is the blowup of the disjoint union of the two components of $\mathbf{M}$ along the singular loci. The two irreducible components of $\mathbf{H}$ are nonsingular. 
This paper is organized as follows. In $\S 2$ and $\S 3$, we classify rational curves in $\mathcal{N}$. In $\S 4$, we study the moduli space of stable maps to a projective space. In $\S 5$, we recall the Hecke correspondence and the partial desingularization $\widetilde{\mathcal{M}}_{X}$. We prove Theorem 1.2 in $\S 6$ and Theorem 1.4 in $\S 7$.

I am most grateful to Professor Michael Thaddeus for sharing his remarkable insight that the partial desingularization $\widetilde{\mathcal{M}}_{X}$ must be the irreducible component of $\overline{\mathbf{M}}_{0,0}(\mathcal{N}, 2)$ containing Hecke curves. I am also grateful to Professor Ravi Vakil for answering a few questions. This paper was written while the author was visiting the mathematics department of Stanford University. It is my pleasure to express gratitude to Professor Jun $\mathrm{Li}$ and the mathematics department of Stanford for generous support and hospitality during my stay.

After finishing this paper, Professor Jun-Muk Hwang kindly informed me of 3] where A.-M. Castravet studied rational curves in $\mathcal{N}$. There seems to be some overlap with her paper and $\S 3$ of this paper. I apologize for any overlap with [3] although our motivations and main results are totally independent.

\section{Examples of Stable maps to $\mathcal{N}$}

In this section we consider various examples of rational curves in the moduli space $\mathcal{N}$ of stable rank 2 bundles with determinant $\mathcal{O}_{X}(-x)$.

2.1. Hecke curves. Let $E \in \mathcal{N}_{X}^{s}$ be a stable rank 2 bundle with $\operatorname{det} E \cong$ $\mathcal{O}_{X}(y-x)$ for some $y \in X$. For $\left.\nu \in \mathbb{P} E\right|_{y} ^{\vee} \cong \mathbb{P}^{1}$, let $E^{\nu}$ denote the kernel of the composition

$$
\left.E \longrightarrow E\right|_{y} \stackrel{\nu}{\longrightarrow} \mathbb{C}
$$

of $\nu$ and the restriction to $y$. Then $E^{\nu}$ is a stable bundle with $\operatorname{det} E^{\nu} \cong$ $\mathcal{O}(-x)$. On $\left.\mathbb{P} E\right|_{y} \times X=\mathbb{P}^{1} \times X$, we have a canonical map

$$
\left.\pi_{X}^{*} E \rightarrow \pi_{X}^{*} E\right|_{y} \rightarrow \mathcal{O}_{\left.\mathbb{P} E\right|_{y} \times\{y\}}(1)
$$

where $\pi_{X}$ denotes the projection onto $X$. The kernel $\mathcal{E}$ of (2.1) is a family of rank 2 bundles on $X$ with determinant $\mathcal{O}(-x)$, parameterized by $\mathbb{P}^{1}$. Therefore we obtain a morphism $f_{E, y}: \mathbb{P}^{1} \rightarrow \mathcal{N}$ since $\mathcal{N}$ is the moduli space of such bundles.

Definition 2.1. For $E \in \mathcal{M}_{X}^{s}$ with $\operatorname{det} E \cong \mathcal{O}_{X}(y-x)$, the induced morphism $f_{E, y}: \mathbb{P}^{1} \rightarrow \mathcal{N}$ is called a Hecke curve.

Conversely, suppose the pull-back $\mathcal{E}$ of a universal bundle on $\mathcal{N} \times X$ to $\mathbb{P}^{1} \times X$ by $f \times i d_{X}$ for a morphism $f: \mathbb{P}^{1} \rightarrow \mathcal{N}$ fits into an exact sequence

$$
0 \rightarrow \mathcal{E} \rightarrow \pi_{X}^{*} E \rightarrow \mathcal{O}_{\mathbb{P}^{1} \times\{y\}}(1) \rightarrow 0 .
$$

Then $f$ is a Hecke curve as it is given by $\left.E\right|_{y} \otimes \mathcal{O}_{\mathbb{P}^{1} \times\{y\}} \rightarrow \mathcal{O}_{\mathbb{P}^{1} \times\{y\}}(1)$ which is unique modulo the action of $P G L(2)$.

Upon restricting (2.2) to $\mathbb{P}^{1} \times\{y\}$ we get a map

$$
\left.\mathcal{E}\right|_{\mathbb{P}^{1} \times\{y\}} \rightarrow \mathcal{O}_{\mathbb{P}^{1} \times\{y\}}(-1) \rightarrow 0
$$


and thus an exact sequence

$$
0 \rightarrow \pi_{X}^{*} E(-y) \rightarrow \mathcal{E} \rightarrow \mathcal{O}_{\mathbb{P}^{1} \times\{y\}}(-1) \rightarrow 0 .
$$

So we obtain the following with $F=E(-y)$.

Lemma 2.2. Let $f: \mathbb{P}^{1} \rightarrow \mathcal{N}$ be a morphism such that the pull-back $\mathcal{E}$ of the universal bundle on $\mathcal{N} \times X$ by $f \times i d_{X}$ fits into an exact sequence

$$
0 \rightarrow \pi_{X}^{*} F \rightarrow \mathcal{E} \rightarrow \mathcal{O}_{\mathbb{P}^{1} \times\{y\}}(-1) \rightarrow 0
$$

for a rank 2 bundle $F$ and $y \in X$. Then $f$ is a Hecke curve.

We recall the following results of Narasimhan and Ramanan.

Theorem 2.3. [13, §5]

(1) For any stable bundle $E$ with $\operatorname{det} E \cong \mathcal{O}_{X}(y-x), f_{E, y}$ is an embedding and the image is a smooth rational curve in $\mathcal{N}$ of degree 2.

(2) The normal bundle of a Hecke curve is generated by global sections.

(3) For distinct $E \in \mathcal{M}_{X}^{s}$, the corresponding Hecke curves are distinct.

Proof. We only provide a proof of (3). Let $f: \mathbb{P}^{1} \rightarrow \mathcal{N}$ be a Hecke curve and let $\mathcal{E} \rightarrow \mathbb{P}^{1} \times X$ be the pull-back of a universal bundle over $\mathcal{N} \times X$ such that $\left.\mathcal{E}\right|_{\mathbb{P}^{1} \times z} \cong \mathcal{O} \oplus \mathcal{O}$ for general $z \in X$. Then $E=\pi_{X *} \mathcal{E} \otimes \mathcal{O}_{X}(y) \in \mathcal{M}_{X}^{s}$ is the unique rank 2 bundle which gives us the Hecke curve $f$ by Theorem 3.2 and (2.3).

Remark 2.4. Item (2) implies that $H^{1}\left(\mathbb{P}^{1}, f^{*} T \mathcal{N}\right)=0$ and hence $\overline{\mathbf{M}}_{0,0}(\mathcal{N}, 2)$ is nonsingular at the points of Hecke curves.

With the Hecke curves, we can generate many more rational curves of higher degrees. First, we can compose a degree $l$ map $\mathbb{P}^{1} \rightarrow \mathbb{P}^{1}$ with a Hecke curve $f_{E, y}: \mathbb{P}^{1} \rightarrow \mathcal{N}$ and get a degree $2 l$ map $f: \mathbb{P}^{1} \rightarrow \mathcal{N}$. In this case the pull-back $\mathcal{E}$ of the universal bundle fits into an exact sequence

$$
0 \rightarrow \pi_{X}^{*} F \rightarrow \mathcal{E} \rightarrow \mathcal{O}_{\mathbb{P}^{1} \times\{y\}}(-l) \rightarrow 0
$$

where $F=E(-y)$. Conversely, if a morphism $f: \mathbb{P}^{1} \rightarrow \mathcal{N}$ gives us (2.5), it should be the composition of a Hecke curve with a degree $l$ covering $\mathbb{P}^{1} \rightarrow \mathbb{P}^{1}$.

More generally, let $l_{1}, \cdots, l_{r}$ be a sequence of positive integers and $y_{1}, \cdots, y_{r}$ be distinct points in $X$. Let $E$ be a rank 2 bundle on $X$ with $\operatorname{det} E \cong$ $\mathcal{O}\left(y_{1}+\cdots+y_{r}-x\right)$ such that

$$
\operatorname{deg} L<\frac{1}{2}(\operatorname{deg} E-r) \text { for any line subbundle } L \text {. }
$$

Over $\left.\mathbb{P} E\right|_{y_{1}} ^{\vee} \times \cdots \times\left.\mathbb{P} E\right|_{y_{r}} ^{\vee} \times X$, we have a canonical map

$$
\left.\left.\pi_{X}^{*} E \rightarrow \pi_{X}^{*} E\right|_{y_{1}} \oplus \cdots \oplus \pi_{X}^{*} E\right|_{y_{r}} \rightarrow \mathcal{O}_{\left.\mathbb{P} E\right|_{y_{1}} ^{\vee} \times\left\{y_{1}\right\}}(1) \oplus \cdots \oplus \mathcal{O}_{\left.\mathbb{P} E\right|_{y_{r}} ^{\vee} \times\left\{y_{r}\right\}}(1)
$$

whose kernel is a family of stable bundles with determinant $\mathcal{O}_{X}(-x)$. Thus we have a morphism

$$
\left.\mathbb{P} E\right|_{y_{1}} ^{\vee} \times \cdots \times\left.\mathbb{P} E\right|_{y_{r}} ^{\vee} \rightarrow \mathcal{N}
$$


Now degree $l_{i}$ maps $\left.\mathbb{P}^{1} \rightarrow \mathbb{P} E\right|_{y_{i}} ^{\vee}$ give us a map

$$
f:\left.\mathbb{P}^{1} \rightarrow \mathbb{P} E\right|_{y_{1}} ^{\vee} \times \cdots \times\left.\mathbb{P} E\right|_{y_{r}} ^{\vee} \rightarrow \mathcal{N}
$$

whose degree is $2\left(l_{1}+\cdots+l_{r}\right)$. Let us call this a generalized Hecke curve. The pull-back $\mathcal{E}$ of a universal bundle fits into an exact sequence

$$
0 \rightarrow \pi_{X} F \rightarrow \mathcal{E} \rightarrow \oplus_{i} \mathcal{O}_{\left.\mathbb{P} E\right|_{y_{i}} ^{\vee} \times\left\{y_{i}\right\}}\left(-l_{i}\right) \rightarrow 0
$$

where $F=E\left(-y_{1}-\cdots-y_{r}\right)$. Conversely, if $f: \mathbb{P}^{1} \rightarrow \mathcal{N}$ induces $\mathcal{E}$ given by (2.7), then $f$ is a generalized Hecke curve.

Remark 2.5. (2.6) requires that every elementary modification by an element of $\left.\mathbb{P} E\right|_{y_{1}} ^{\vee} \times \cdots \times\left.\mathbb{P} E\right|_{y_{r}} ^{\vee}$ be stable. But to get a generalized Hecke curve we only have to require that the elementary modifications by elements in the image of $f$ be stable.

2.2. Extensions of line bundles. A different way of producing rational curves in $\mathcal{N}$ is to consider extension bundles. For $\xi \in \operatorname{Pic}^{k}(X)$, let $\mathbb{P} \operatorname{Ext}^{1}\left(\xi, \xi^{-1}(-x)\right)^{s}=\mathbb{P} H^{1}\left(\xi^{-2}(-x)\right)^{s}$ be the locus of extension bundles

$$
0 \rightarrow \xi^{-1}(-x) \rightarrow E \rightarrow \xi \rightarrow 0
$$

which are stable. Then we have an obvious morphism

$$
\theta_{\xi}: \mathbb{P} \operatorname{Ext}^{1}\left(\xi, \xi^{-1}(-x)\right)^{s} \rightarrow \mathcal{N} .
$$

Suppose $f^{\prime}: \mathbb{P}^{1} \rightarrow \mathbb{P E x t}\left(\xi, \xi^{-1}(-x)\right)$ is a morphism of degree $a$ whose image lies in $\mathbb{P} E x t^{1}\left(\xi, \xi^{-1}(-x)\right)^{s}$. Then the composition of $f^{\prime}$ with $\theta_{\xi}$ gives us a stable map $f: \mathbb{P}^{1} \rightarrow \mathcal{N}$.

When $k=0$ it is easy to check that $\mathbb{P} \operatorname{Ext}^{1}\left(\xi, \xi^{-1}(-x)\right)^{s}=\mathbb{P} E x t^{1}\left(\xi, \xi^{-1}(-x)\right)$. We recall the following.

Proposition 2.6. [13, 6.18] $\theta_{\xi}$ is an embedding of degree 1 for any $\xi \in$ $\operatorname{Pic}{ }^{0}(X)$, i.e. the pull-back of the ample generator of $\operatorname{Pic}(\mathcal{N})$ is $\mathcal{O}(1)$.

In general, we have the following.

Lemma 2.7. For $\xi \in P i c^{k}(X)$, the degree of $\theta_{\xi}$ is $2 k+1$.

Proof. The universal extension bundle $\mathcal{U}$ over $\mathbb{P} E x t^{1}\left(\xi, \xi^{-1}(-x)\right)^{s} \times X$ fits into an exact sequence

$$
0 \rightarrow \pi_{X}^{*} \xi^{-1}(-x) \otimes \mathcal{O}_{\mathbb{P}}(1) \rightarrow \mathcal{U} \rightarrow \pi_{X}^{*} \xi \rightarrow 0
$$

By [13, 6.7], the tangent bundle $T \mathbb{P}$ of $\mathbb{P} E x t^{1}\left(\xi, \xi^{-1}(-x)\right)^{s}$ fits into an exact sequence

$$
0 \rightarrow T \mathbb{P} \rightarrow R^{1} \pi_{\mathbb{P}_{*}}\left(\mathcal{U} \otimes \pi_{X}^{*} \xi^{-1}\right) \rightarrow R^{1} \pi_{\mathbb{P}_{*}} \mathcal{O}=H^{1}(X, \mathcal{O}) \otimes \mathcal{O}_{\mathbb{P}} \rightarrow 0
$$

where $\pi_{\mathbb{P}}$ denotes the projection onto $\mathbb{P} E x t^{1}\left(\xi, \xi^{-1}(-x)\right)^{s}$. Since

$$
\operatorname{dim} \mathbb{P} \operatorname{Ext}^{1}\left(\xi, \xi^{-1}(-x)\right)^{s}=2 k+g-1
$$

by Riemann-Roch,

$$
c_{1}\left(R^{1} \pi_{\mathbb{P} *}\left(\mathcal{U} \otimes \pi_{X}^{*} \xi^{-1}\right)\right)=c_{1}(T \mathbb{P})=\mathcal{O}(2 k+g) .
$$


Also we have an exact sequence

$$
0 \rightarrow \mathcal{U} \otimes \pi_{X}^{*} \xi^{-1} \rightarrow E n d_{0} \mathcal{U} \rightarrow \pi_{X}^{*} \xi^{2}(x) \otimes \mathcal{O}_{\mathbb{P}}(-1) \rightarrow 0
$$

which gives rise to an exact sequence

$0 \rightarrow H^{0}\left(\xi^{2}(x)\right) \otimes \mathcal{O}_{\mathbb{P}}(-1) \rightarrow R^{1} \pi_{\mathbb{P}_{*}}\left(\mathcal{U} \otimes \pi_{X}^{*} \xi^{-1}\right) \rightarrow R^{1} \pi_{\mathbb{P}_{*}} \operatorname{End}_{0} \mathcal{U} \rightarrow H^{1}\left(\xi^{2}(x)\right) \otimes \mathcal{O}_{\mathbb{P}}(-1) \rightarrow 0$.

By Riemann-Roch,

$\operatorname{det}\left(H^{0}\left(\xi^{2}(x)\right) \otimes \mathcal{O}_{\mathbb{P}}(-1)\right)^{-1} \otimes \operatorname{det}\left(H^{1}\left(\xi^{2}(x)\right) \otimes \mathcal{O}_{\mathbb{P}}(-1)\right) \cong \mathcal{O}_{\mathbb{P}}(2 k-g+2)$

Since $\theta_{\xi}^{*} T \mathcal{N}=R^{1} \pi_{\mathbb{P}_{*}} E n d_{0} \mathcal{U}, \operatorname{deg}\left(f^{*} T \mathcal{N}\right)=(2 k-g+2)+(2 k+g)=4 k+2$. Because $c_{1}(T \mathcal{N})$ is 2 times the ample generator of $\operatorname{Pic}(\mathcal{N})$, the degree of $f$ is $2 k+1$.

As a consequence, a degree $a \operatorname{map} f^{\prime}: \mathbb{P}^{1} \rightarrow \mathbb{P} E x t^{1}\left(\xi, \xi^{-1}(-x)\right)^{s}$ for some $\xi \in P i c^{k}(X)$ composed with $\theta_{\xi}$ is a map $f: \mathbb{P}^{1} \rightarrow \mathcal{N}$ of degree $a(2 k+1)$. The pull-back of a universal bundle on $\mathcal{N} \times X$ by $f \times i d_{X}$ embeds into an exact sequence

$$
0 \rightarrow \pi_{X}^{*} \xi^{-1}(-x) \otimes \mathcal{O}_{\mathbb{P}}(a) \rightarrow \mathcal{E} \rightarrow \pi_{X}^{*} \xi \rightarrow 0 .
$$

Conversely, it is obvious that any such $\mathcal{E}$ is given by a morphism

$$
f: \mathbb{P}^{1} \rightarrow \mathbb{P} \operatorname{Ext}^{1}\left(\xi, \xi^{-1}(-x)\right)^{s} \rightarrow \mathcal{N} .
$$

More generally, if we have a rational map $f^{\prime}: \mathbb{P}^{1} \rightarrow \mathbb{P} E x t^{1}\left(\xi, \xi^{-1}(-x)\right)^{s}$ of degree $a$, the composition of $f^{\prime}$ with $\theta_{\xi}$ uniquely extends to a morphism $f: \mathbb{P}^{1} \rightarrow \mathcal{N}$. The pull-back $\mathcal{E}$ of the universal bundle on $\mathcal{N} \times X$ now embeds into an exact sequence

$$
0 \rightarrow \pi_{X}^{*} \xi^{-1}(-x) \otimes \mathcal{O}_{\mathbb{P}}(a) \rightarrow \mathcal{E} \rightarrow \pi_{X}^{*} \xi \otimes \mathcal{J}_{Z} \rightarrow 0
$$

for a zero-dimensional subscheme $Z$ of $\mathbb{P}^{1} \times X$. (See Theorem 3.2 (2).)

\section{Classification of Rational CuRves in $\mathcal{N}$}

In this section we show that the examples discussed in $\S 3$ are all possible nonconstant maps $f: \mathbb{P}^{1} \rightarrow \mathcal{N}$.

Suppose $f: \mathbb{P}^{1} \rightarrow \mathcal{N}$ is a nonconstant morphism. The pull-back of a universal bundle over $\mathcal{N} \times X$ by $f \times i d_{X}$ is a rank 2 bundle

$$
\mathcal{E} \rightarrow \mathbb{P}^{1} \times X=: S
$$

As before, let $\pi_{X}, \pi_{P}$ denote the projections of $S$ to $X$ and $\mathbb{P}^{1}$ respectively. The starting point of our classification is the following lemma due to X. Sun.

Lemma 3.1. 14, §2] Let $f: \mathbb{P}^{1} \rightarrow \mathcal{N}$ be a morphism of degree $\beta \in \mathbb{Z}_{>0}$ and $\mathcal{E}$ be the induced vector bundle. Then

$$
\beta=2 c_{2}(\varepsilon)-\frac{1}{2} c_{1}(\mathcal{E})^{2} .
$$


Proof. It is well-known that $f^{*} T \mathcal{N}=R^{1} \pi_{P *} E n d_{0} \mathcal{E}$ and $\pi_{P *} E n d_{0} \mathcal{E}=0$ by stability where $E n d_{0} \mathcal{E}$ is the traceless part of the endomorphism bundle of $\mathcal{E}$. By Leray's spectral sequence and Riemann-Roch, we have

$$
\chi\left(S, E n d_{0} \mathcal{E}\right)=-\chi\left(\mathbb{P}^{1}, f^{*} T \mathcal{N}\right)=-(3 g-3+2 \beta)
$$

since $\operatorname{deg} c_{1}\left(f^{*} T \mathcal{N}\right)=\operatorname{deg} f^{*} c_{1}(T \mathcal{N})=2 \beta$. On the other hand, by RiemannRoch for $S$ we have

$$
\chi\left(S, E n d_{0} \mathcal{E}\right)=\int_{S} \operatorname{ch}\left(E n d_{0} \mathcal{E}\right) \cdot t d_{X} \cdot t d_{\mathbb{P}^{1}}=-3(g-1)-c_{2}\left(E n d_{0} \mathcal{E}\right) .
$$

Therefore $\beta=\frac{1}{2} c_{2}\left(E n d_{0} \mathcal{E}\right)=2 c_{2}(\mathcal{E})-\frac{1}{2} c_{1}(\mathcal{E})^{2}$.

By tensoring with $\pi_{P}^{*} \mathcal{O}(d)$ for suitable $d \in \mathbb{Z}$ we may assume that $\left.\mathcal{E}\right|_{\mathbb{P}^{1} \times\{z\}}$ for general $z$ is $\mathcal{O}_{\mathbb{P}^{1}}(a) \oplus \mathcal{O}_{\mathbb{P}^{1}}$ for some $a \geq 0$. This doesn't change the morphism $f$ since tensoring by $\pi_{P}^{*} \mathcal{O}(d)$ gives us an equivalent family of stable bundles. We recall the following result of Brosius.

Theorem 3.2. 1, Theorem 1] Let $\mathcal{E}$ be as above. Then $\mathcal{E}$ belongs to an exact sequence

$$
0 \rightarrow \mathcal{E}^{\prime} \rightarrow \mathcal{E} \rightarrow \mathcal{E}^{\prime \prime} \rightarrow 0
$$

such that the following hold.

(1) If $a=0$, there exist a rank 2 bundle $F$ on $X$ and $Z \in \operatorname{Hilb}^{l}(S)$ for some $l \geq 0$ such that $\mathcal{E}^{\prime}=\pi_{X}^{*} F$ and $\mathcal{E}^{\prime \prime}=\mathfrak{J}_{Z \subset Y}$ is the ideal sheaf of $Z$ in $Y=\pi_{X}^{-1} \pi_{X}(Z)$.

(2) If $a>0$, there exist a line bundle $\xi \in P i c^{k}(X)$ for some $k \geq 0$ and $Z \in \operatorname{Hilb}^{l}(S)$ for some $l \geq 0$ such that $\mathcal{E}^{\prime}=\pi_{X}^{*} \xi^{-1}(-x) \otimes \pi_{P}^{*} \mathcal{O}_{\mathbb{P}^{1}}(a)$ and $\mathcal{E}^{\prime \prime}=\mathcal{J}_{Z} \otimes \pi_{X}^{*} \xi$.

Furthermore, $\mathcal{E}^{\prime}$ and $\mathcal{E}^{\prime \prime}$ are unique up to isomorphism.

Definition 3.3. We say $f$ is of Hecke type when (1) holds and $f$ is of extension type when (2) holds.

Remark 3.4. (1) Suppose $f$ is of Hecke type. If $l=1, f$ is a Hecke curve by (2.4) because $\mathcal{J}_{Z \subset Y} \cong \mathcal{O}_{\mathbb{P}^{1} \times\{y\}}(-1)$ for $y=\pi_{X}(Z)$. When $l>1, f$ is a generalized Hecke curve by (2.7).

(2) Any extension

$$
0 \rightarrow \pi_{X}^{*} \xi^{-1}(-x) \otimes \pi_{P}^{*} \mathcal{O}_{\mathbb{P}^{1}}(a) \rightarrow \mathcal{E} \rightarrow \mathcal{J}_{Z} \otimes \pi_{X}^{*} \xi \rightarrow 0
$$

is locally free since the Cayley-Bacharach property is satisfied ([5] Theorem 5.1.1]) from the vanishing

$H^{0}\left(\pi_{X}^{*} K_{X} \otimes \pi_{X}^{*} \xi^{2}(x) \otimes \pi_{P}^{*} \mathcal{O}(-a) \otimes \pi_{P}^{*} K_{\mathbb{P}^{1}}\right)=H^{0}\left(X, K_{X} \otimes \xi^{2}(x)\right) \otimes H^{0}\left(\mathbb{P}^{1}, \mathcal{O}(-a-2)\right)=0$.

(3) If $f$ is of extension type and $l=0$, then $f$ is a line in $\mathbb{P} H^{1}\left(\xi^{-2}(-x)\right)^{s}$. When $l>0, f$ is given by a rational map $\mathbb{P}^{1} \rightarrow \mathbb{P} H^{1}\left(\xi^{-2}(-x)\right)^{s}$ of degree $a$.

By direct computation with Lemma 3.1, we obtain the following useful lemma. 
Lemma 3.5. With notation as in Theorem 3.2, $\beta=2 l$ for the Hecke type maps and $\beta=2 l+a(2 \operatorname{deg} \xi+1)$ for the extension type maps.

We can now classify rational curves of degree $\leq 2$ in $\mathcal{N}$.

Proposition 3.6. Let $f: \mathbb{P}^{1} \rightarrow \mathcal{N}$ is of degree $\leq 2$. Then $f$ is one of the following.

(1) $f$ is a Hecke curve for some $y \in X$ and a stable rank 2 bundle $E$ with determinant $\mathcal{O}_{X}(y-x)$

(2) $f$ is a conic in $\mathbb{P} H^{1}\left(\xi^{-2}(-x)\right)$ for some $\xi \in P i c^{0}(X)$

(3) $f$ is a line in $\mathbb{P} H^{1}\left(\xi^{-2}(-x)\right)$ for some $\xi \in P i c^{0}(X)$.

The degree of $f$ is 2 for (1) and (2) while 1 for (3).

Proof. By Lemma 3.5] $\beta=1$ is possible only when $f$ is of extension type with $l=0, a=1, \operatorname{deg} \xi=0$. This holds exactly when (3) above holds. Suppose $\beta=2$ now. If $f$ is of Hecke type, then $l=1$ which means $f$ is a Hecke curve. If $f$ is of extension type, then $l=0, a=2$ and $\operatorname{deg} \xi=0$ which means that $f$ is a conic in $\mathbb{P} H^{1}\left(\xi^{-2}(-x)\right)$.

As a first consequence, we obtain the moduli space $\overline{\mathbf{M}}_{0,0}(\mathcal{N}, 1)$ of stable maps of degree 1 .

Theorem 3.7. 12, 14 Let $\mathcal{L} \rightarrow \operatorname{Pic}^{0}(X) \times X$ be a Poincaré bundle and let $\pi: \operatorname{Pic}^{0}(X) \times X \rightarrow \operatorname{Pic}^{0}(X)$ be the projection. Let

$$
\operatorname{Gr}(2, \mathcal{W}) \rightarrow \operatorname{Pic}^{0}(X)=J
$$

be the Grassmannian bundle of 2 dimensional subspaces of the bundle

$$
\mathcal{W}=R^{1} \pi_{*} \mathcal{L}^{-2}(-x)=R^{1} \pi_{*} \operatorname{Hom}\left(\mathcal{L}, \mathcal{L}^{-1}(-x)\right)
$$

of rank $g$ over $J$. Then we have an isomorphism

$$
\overline{\mathbf{M}}_{0,0}(\mathcal{N}, 1) \cong G r(2, \mathcal{W}) .
$$

Proof. Let $f \in \overline{\mathbf{M}}_{0,0}(\mathcal{N}, 1)$. By Proposition [3.6] $f$ factors through a degree 1 map $\mathbb{P}^{1} \rightarrow \mathbb{P} H^{1}\left(\xi^{-2}(-x)\right)$. Obviously the Grassmannian bundle $G r(2, \mathcal{W})$ parameterizes all such maps and thus we get a natural map

$$
\gamma: \operatorname{Gr}(2, \mathcal{W}) \rightarrow \overline{\mathbf{M}}_{0,0}(\mathcal{N}, 1)
$$

which is bijective by Proposition [2.6 and Proposition [4.6 For a degree 1 extension type map $f$, we will see in $\S 6$ (Proposition 6.3) that

$$
f^{*} T \mathcal{N} \cong \mathcal{O}(2) \oplus \mathcal{O}(1)^{k} \oplus \mathcal{O}(-1)^{k} \oplus \mathcal{O}^{l}
$$

for $k, l$ such that $2 k+l+1=3 g-3$. Therefore $H^{1}\left(\mathbb{P}^{1}, f^{*} T \mathcal{N}\right)=0$ and thus $\overline{\mathbf{M}}_{0,0}(\mathcal{N}, 1)$ is normal. To conclude that $\gamma$ is an isomorphism by Zariski's main theorem, it suffices to show that $\gamma$ is birational. Locally in usual topology on the smooth part, $\gamma$ is given by holomorphic maps $\gamma: \mathbb{C}^{n} \rightarrow \mathbb{C}^{n}$. If the Jacobian determinant is nonzero at some point, then $\gamma$ is birational. It is an elementary consequence of inverse function theorem that if the Jacobian determinant is everywhere zero the map cannot be one to one. So we are done. 
Note that $\operatorname{Gr}(2, \mathcal{W})$ is the moduli space of relative stable maps $\overline{\mathbf{M}}_{0,0}(\mathbb{P W} / J, 1)$.

The open dense subset in $\overline{\mathbf{M}}_{0,0}(\mathcal{N}, 2)$ of irreducible curves consists of stable maps given in (1) and (2) of Proposition 3.6. In $\S 6$, we will prove that $\overline{\mathbf{M}}_{0,0}(\mathcal{N}, 2)$ consists of two irreducible components: Hecke curves will give us an irreducible component of dimension $3 g-2$ (isomorphic to the partial desingularization of $\mathcal{M}_{X}$ ) and the extension type maps will give us an irreducible component of dimension $4 g-4$ (isomorphic to $\widetilde{Q}_{J}=\overline{\mathbf{M}}_{0,0}(\mathbb{P} \mathcal{W} / J, 2)$ in $\S 4$ ).

When the degree of $f$ is 3 , Lemma 3.5] says that $f$ has to be of extension type and there are three possibilities:

(1) $l=0, a=3, \operatorname{deg} \xi=0$

(2) $l=0, a=1, \operatorname{deg} \xi=1$

(3) $l=1, a=1, \operatorname{deg} \xi=0$.

The first case is a cubic curve in $\mathbb{P} E x t^{1}\left(\xi, \xi^{-1}(-x)\right)=\mathbb{P} H^{1}\left(\xi^{-2}(-x)\right)$ for $\xi \in P i c^{0}(X)$ and the second case is a line in $\mathbb{P} H^{1}\left(\xi^{-2}(-x)\right)^{s}$ for $\xi \in P i c^{1}(X)$. We claim the third case is impossible.

Lemma 3.8. Suppose $f$ is of extension type and $\operatorname{deg} \xi=0$. Then $l=0$.

Proof. Suppose $l>0$ and let $(p, y) \in \operatorname{Supp} Z \subset \mathbb{P}^{1} \times X$. When $\operatorname{deg} \xi=0$, it is easy to see that the restriction of $\mathcal{E}$ to $\{p\} \times X$ is unstable. Hence this is impossible.

The following classification of maps $f: \mathbb{P}^{1} \rightarrow \mathcal{N}$ of degree 3 and 4 is direct from Lemmas 3.5 and 3.8 .

Proposition 3.9. (1) A degree 3 map $f: \mathbb{P}^{1} \rightarrow \mathcal{N}$ comes from either a cubic curve in $\mathbb{P} H^{1}\left(\xi^{-2}(-x)\right)$ for some $\xi \in P i c^{0}(X)$ or a line in $\mathbb{P} H^{1}\left(\xi^{-2}(-x)\right)^{s}$ for some $\xi \in P i c^{1}(X)$.

(2) A degree 4 map $f: \mathbb{P}^{1} \rightarrow \mathcal{N}$ comes from either a quartic curve in $\mathbb{P} H^{1}\left(\xi^{-2}(-x)\right)$ for some $\xi \in P i c^{0}(X)$ or a double cover of a Hecke curve.

\section{Stable maps to Projective spaces}

In this section we describe the moduli space $\overline{\mathbf{M}}_{0,0}\left(\mathbb{P}^{g-1}, 2\right)$ of stable maps of degree 2 in $\mathbb{P}^{g-1}$ as the partial desingularization of a GIT quotient.

Let $V$ be a vector space of dimension $g$ and let $f: \mathbb{P}^{1} \rightarrow \mathbb{P} V=\mathbb{P}^{g-1}$ be a degree 2 embedding. Then we have a surjection

$$
V^{\vee}=H^{0}(\mathbb{P} V, \mathcal{O}(1)) \rightarrow H^{0}\left(\mathbb{P}^{1}, \mathcal{O}(2)\right)=H^{0}\left(T \mathbb{P}^{1}\right) \cong s l(2)
$$

because $H^{0}\left(T \mathbb{P}^{1}\right)=\operatorname{Lie} A u t\left(\mathbb{P}^{1}\right)=\operatorname{sl}(2)$. Conversely, any such surjection gives us an embedding of degree 2 . Hence the moduli space of degree 2 embeddings of $\mathbb{P}^{1}$ in $\mathbb{P} V$ is the quotient

$$
\mathbb{P H o m}_{3}\left(s l(2)^{\vee}, V\right) / P G L(2)
$$


where the subscript 3 denotes the open locus of rank 3 homomorphisms. The compactification $\overline{\mathbf{M}}_{0,0}(\mathbb{P} V, 2)$ turns out to be Kirwan's partial desingularization of the GIT quotient ([9])

$$
Q:=\mathbb{P} \operatorname{Hom}\left(s l(2)^{\vee}, V\right) / / P G L(2) .
$$

Theorem 4.1. Let $P_{1}=\mathbb{P H o m}_{1}\left(\operatorname{sl}(2)^{\vee}, V\right)$ denote the locus of rank 1 homomorphisms in $P:=\mathbb{P H o m}\left(\operatorname{sl}(2)^{\vee}, V\right)$. Let $\widetilde{P}$ be the blowup of $P$ along $P_{1}$ and let $\widetilde{P}^{s}$ be the set of stable points in $\widetilde{P}$ with respect to a linearization close to the pull-back of $\mathcal{O}_{P}(1)$ [9, §3]. Then we have an $S L(2)$-invariant morphism

$$
\phi: \widetilde{P}^{s} \rightarrow \overline{\mathbf{M}}_{0,0}(\mathbb{P} V, 2)
$$

which induces an isomorphism

$$
\widetilde{Q}:=\widetilde{P}^{s} / P G L(2) \stackrel{\cong}{\longrightarrow} \overline{\mathbf{M}}_{0,0}(\mathbb{P} V, 2) .
$$

Remark 4.2. $P_{1}^{s s}$ is the locus of points in $P^{s s}$ whose infinitesimal stabilizer is nontrivial. Hence $\widetilde{Q}=\widetilde{P}^{s} / P G L(2)$ is the partial desingularization defined by Kirwan in 9 .

Proof of Theorem 4.1. To obtain $\phi$, we construct a family of stable maps to $\mathbb{P} V$ parameterized by $\widetilde{P}^{s}$. The evaluation map

$$
\operatorname{sl}(2)^{\vee} \otimes \operatorname{Hom}\left(\operatorname{sl}(2)^{\vee}, V\right) \rightarrow V
$$

gives rise to

$$
H^{0}\left(\mathbb{P}^{1} \times P, \mathcal{O}(1,1)\right)=\operatorname{sl}(2) \otimes \operatorname{Hom}\left(s l(2)^{\vee}, V\right)^{\vee} \leftarrow V^{\vee}=H^{0}(\mathbb{P} V, \mathcal{O}(1)) .
$$

Hence we have a rational map

$$
\Phi: \mathbb{P}^{1} \times \widetilde{P}^{s} \rightarrow \mathbb{P}^{1} \times P \rightarrow \mathbb{P} V
$$

which is a morphism on $\mathbb{P}^{1} \times P_{3}$ where $P_{3}=\mathbb{P H o m}_{3}\left(s l(2)^{\vee}, V\right)$. Let us look at the exceptional points of $\Phi$.

Let $\nu$ be a semistable point in $P$. Obviously, when the rank of $\nu$ is 3 , there is no exceptional point. When the rank of $\nu$ is 2 , let $(0, \nu) \in \mathbb{P}^{1} \times P^{s s}$ be an exceptional point. Then we can choose a basis $\{t, s\}$ of $H^{0}\left(\mathbb{P}^{1}, \mathcal{O}(1)\right)$ such that the image of $\nu$ is the span of $t^{2}$ and $t s$. But then $\nu$ is a strictly semistable point whose orbit closure intersects with $P_{1}$ and thus $\nu$ becomes unstable after blowing up $P$ along $P_{1}[9, \S 6]$. Hence such $\nu$ is discarded after the blow-up.

Now suppose the rank of $\nu$ is 1 . Then there are two exceptional points of the rational map $\mathbb{P}^{1} \rightarrow \mathbb{P} V$ induced by $\nu$. But when the two points coincide, $\nu$ becomes unstable after the blowup along $P_{1}$ by the same reason as in the previous paragraph and hence is discarded. Therefore, the exceptional locus of $\Phi$ consists of two disjoint sections $s_{1}, s_{2}$ over $\widetilde{P}_{1}^{s}$, the inverse image of $P_{1}$ in $\widetilde{P}^{s}$. In particular, the exceptional locus consists of two codimension 2 
subvarieties. We blow up $\mathbb{P}^{1} \times \widetilde{P}^{s}$ along the exceptional locus and denote the blowup map by

$$
\Gamma \stackrel{\rho}{\longrightarrow} \mathbb{P}^{1} \times \widetilde{P}^{s} \stackrel{\Phi}{-} \rightarrow \mathbb{P} V
$$

and let $D_{1}, D_{2}$ be the exceptional divisors. The line bundle

$$
H=\rho^{*} \mathcal{O}_{\mathbb{P}^{1} \times \widetilde{P}^{s}}(1,1) \otimes \mathcal{O}_{\Gamma}\left(-D_{1}-D_{2}\right)
$$

is equipped with a surjective morphism $V^{\vee} \otimes \mathcal{O}_{\Gamma} \rightarrow H$ by easy local computation and hence the rational map (4.2) is a morphism. Therefore, we have a diagram of morphisms

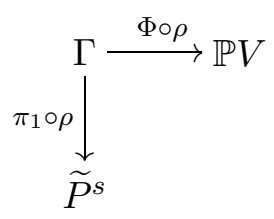

and hence a morphism $\phi: \widetilde{P}^{s} \rightarrow \overline{\mathbf{M}}_{0,0}(\mathbb{P} V, 2)$. From our construction, it is evident that $\phi$ is $P G L(2)$-invariant over the dense open set $P_{3}$ and thus invariant everywhere.

Since $\mathbb{P} V$ is convex, $\overline{\mathbf{M}}_{0,0}(\mathbb{P} V, 2)$ is irreducible and normal. Also by GIT, $\widetilde{Q}$ is projective and hence the induced map $\widetilde{Q} \rightarrow \overline{\mathbf{M}}_{0,0}(\mathbb{P} V, 2)$ is surjective. To prove that it is an isomorphism it suffices to check injectivity. It is easy to see by Luna's slice theorem that the exceptional divisor of $\widetilde{Q} \rightarrow Q$ is a $\mathbb{P}^{g-2} \times_{\mathbb{Z}_{2}} \mathbb{P}^{g-2}$-bundle over $\mathbb{P}^{g-1}$ which is precisely the set of intersecting lines in $\mathbb{P} V$. So we are done.

Note that $\overline{\mathbf{M}}_{0,0}(\mathbb{P} V, 2)=\widetilde{P}^{s} / P G L(2)=\widetilde{Q}$ is singular along the proper transform of the quotient $Q_{2}$ of the locus of rank $\leq 2$ homomorphisms $P_{2}:=\mathbb{P H o m}_{2}\left(s l(2)^{\vee}, V\right)$ by $P G L(2)$. In fact the proper transform of $P_{2}$ in $\widetilde{P}^{s}$ is the locus of nontrivial stabilizers which are isomorphic to $\mathbb{Z}_{2}$. By blowing up along this singular locus we get a (full) desingularization $\widehat{Q}$.

Proposition 4.3. $\widehat{Q}$ is the variety of complete conics $C C(\mathcal{B})$ where $\mathcal{B} \rightarrow$ $\operatorname{Gr}(3, V)$ is the universal bundle over the Grassmannian, i.e. $\widehat{Q}$ is the blowup of $\mathbb{P}\left(S^{2} \mathcal{B}\right)$ along the locus $\mathbb{P}\left(S_{1}^{2} \mathcal{B}\right)$ of rank 1 conics.

Proof. For $\alpha \in \operatorname{Hom}\left(s l(2)^{\vee}, B\right)$ for $B \in G r(3, V)$, consider the composition

$$
B^{\vee} \stackrel{\alpha^{\vee}}{\longrightarrow} \operatorname{sl}(2) \longrightarrow \operatorname{sl}(2)^{\vee} \stackrel{\alpha}{\longrightarrow} B
$$

where the middle map is given by the invariant pairing on $\operatorname{sl}(2)$. This gives rise to an isomorphism

$$
\mathbb{P H o m}\left(\operatorname{sl}(2)^{\vee}, \mathcal{B}\right) / / P G L(2) \rightarrow \mathbb{P}\left(S^{2} \mathcal{B}\right)
$$

and hence an isomorphism of blow-ups

$$
b l_{\mathbb{P} \operatorname{Hom}_{1}\left(s l(2)^{\vee}, \mathcal{B}\right)} \mathbb{P} \operatorname{Hom}\left(\operatorname{sl}(2)^{\vee}, \mathcal{B}\right) / / P G L(2) \cong b l_{\mathbb{P}\left(S_{1}^{2} \mathcal{B}\right)} \mathbb{P}\left(S^{2} \mathcal{B}\right)=C C(\mathcal{B}) .
$$


On the other hand, the inclusion $\mathcal{B} \hookrightarrow V \otimes \mathcal{O}_{G r(3, V)}$ induces a morphism $\mathbb{P H o m}\left(\operatorname{sl}(2)^{\vee}, \mathcal{B}\right) \rightarrow \mathbb{P H o m}\left(\operatorname{sl}(2)^{\vee}, V\right)$ which preserves the loci of rank $\leq i$ homomorphisms for $i=1,2$. Hence we have a map

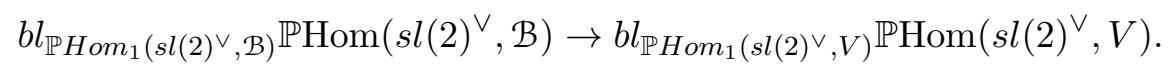

As the proper transform of $\mathbb{P H o m} 2\left(\operatorname{sl}(2)^{\vee}, \mathcal{B}\right)$ is a divisor of the left side, we can further blow up the right side along the proper transform $\widetilde{\mathbb{P H o m}}_{2}\left(s l(2)^{\vee}, V\right)$ of $\mathbb{P H o m}_{2}\left(\operatorname{sl}(2)^{\vee}, V\right)$ and get a morphism

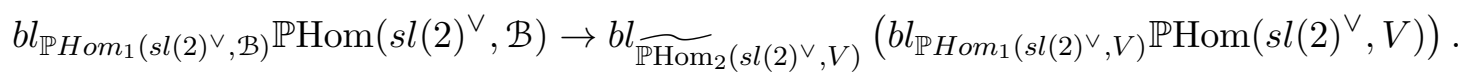

The quotient of the right side is the blowup $\widehat{Q}$ of $\widetilde{Q}$ and hence we obtain a map

$$
\phi: C C(\mathcal{B}) \rightarrow \widehat{Q}
$$

We claim this is bijective and therefore an isomorphism. Since every conic uniquely determines a projective plane $\mathbb{P} B$ containing it unless it is a double line, $\phi$ is a bijective over the complement in $\widetilde{Q}$ of the locus of double lines. Now from the proof of Theorem 4.1 the locus of the double lines in $\widetilde{Q}$ is exactly $\widetilde{\mathbb{P H o m}}_{2}\left(\operatorname{sl}(2)^{\vee}, V\right) / / P G L(2)$ and the normal cones are $\mathbb{C}^{g-2} / \mathbb{Z}_{2}$ by Luna's slice theorem. Hence the fibers of $\widehat{Q} \rightarrow \widetilde{Q}$ over double lines are $\mathbb{P}^{g-3}$ which parameterizes choices of 3-dimensional subspaces of $V$ containing the image of $s l(2)^{\vee}$. The fibers of $C C(\mathcal{B}) \rightarrow \widetilde{Q}$ over a double line corresponds to choices of a projective plane $\mathbb{P} B$ containing the line and thus $\mathbb{P}^{g-3}$. So $\phi$ is an isomorphism.

We have blowup maps

$$
\mathbb{P}\left(S^{2} \mathcal{B}\right) \stackrel{\Phi_{\mathcal{B}}}{\longleftarrow} C C(\mathcal{B}) \stackrel{\Phi_{\mathcal{B}} \vee}{\longrightarrow} \mathbb{P}\left(S^{2} \mathcal{B}^{\vee}\right)
$$

Let $h_{1}$ be the pull-back to $C C(\mathcal{B})$ of the ample generator $c_{1}\left(\mathcal{B}^{\vee}\right)$ of $\operatorname{Pic}(G r(3, V))$; let $h_{2}$ be the pull-back to $C C(\mathcal{B})$ of $c_{1}\left(\mathcal{O}_{\mathbb{P}\left(S^{2} \mathcal{B}\right)}(1)\right)$; let $h_{3}$ be the class of the exceptional divisor of $\Phi_{\mathcal{B}}$. Then the Picard group of $C C(\mathcal{B})$ is generated by $h_{1}, h_{2}, h_{3}$.

The exceptional divisor $\widehat{\Delta}$ of the blowup $\widehat{Q}=C C(\mathcal{B}) \rightarrow \mathbb{P}\left(S^{2} \mathcal{B}^{\vee}\right)$ is $\mathbb{P}\left(S^{2}\left(\mathcal{B}^{\vee} / \mathcal{O}_{\mathbb{P} \mathcal{B}} \vee(-1)\right)\right)$ over $\mathbb{P B}^{\vee} \cong \mathbb{P}\left(S_{1}^{2} \mathcal{B}^{\vee}\right)$. Let $\mathcal{A} \rightarrow G r(2, V)$ be the universal bundle. Then we have a natural correspondence

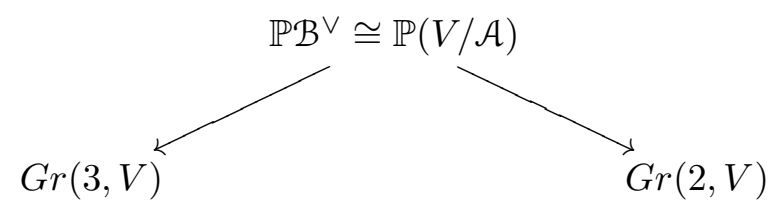

As a bundle over $\mathbb{P}(V / \mathcal{A}), \mathbb{P}\left(S^{2}\left(\mathcal{B}^{\vee} / \mathcal{O}_{\mathbb{P} \mathcal{B} \vee}(-1)\right)\right)$ is the pull-back of $\mathbb{P}\left(S^{2} \mathcal{A}\right)$ and hence

$$
\widehat{\Delta} \cong \mathbb{P}\left(S^{2} \mathcal{A}^{\vee}\right) \times_{G r(2, V)} \mathbb{P}(V / \mathcal{A})
$$


Let $\sigma_{1}$ (resp. $\left.\sigma_{2}\right)$ be the numerical class of a line in a fiber of $\mathbb{P}\left(S^{2} \mathcal{A}^{\vee}\right)$ (resp. $\mathbb{P}(V / \mathcal{A}))$ over $\operatorname{Gr}(2, V)$. Let $\sigma_{3}$ be the numerical class of a line in a fiber of $\Phi_{\mathcal{B}}$. Then it is straightforward to compute the pairing of $h_{i} \cdot \sigma_{j}$ for $i, j=1,2,3$ and obtain the following table:

$\begin{array}{cccc} & h_{1} & h_{2} & h_{3} \\ \sigma_{1} & 0 & 1 & 2 \\ \sigma_{2} & 1 & 0 & 0 \\ \sigma_{3} & 0 & 0 & -1\end{array}$

Also by direct computation the numerical class of $\widehat{\Delta}$ is $-2 h_{1}+3 h_{2}-2 h_{3}$. Hence we have

$$
\sigma_{1} \cdot \widehat{\Delta}=-1, \quad \sigma_{2} \cdot \widehat{\Delta}=-2, \quad \sigma_{3} \cdot \widehat{\Delta}=2
$$

As a consequence the restriction of $\mathcal{O}_{C C(\mathcal{B})}(\widehat{\Delta})$ to a fiber $\mathbb{P}^{2} \times \mathbb{P}^{g-3}$ of $\widehat{\Delta} \rightarrow$ $G r(2, V)$ is $\mathcal{O}(-1,-2)$. The contraction of $C C(\mathcal{B})$ along $\mathbb{P}\left(S^{2} \mathcal{A}^{\vee}\right)$ of $\widehat{\Delta}$ is $\mathbb{P}\left(S^{2} \mathcal{B}^{\vee}\right)$ and the contraction along $\mathbb{P}(V / \mathcal{A})$ is $\widetilde{Q}$. We can further contract $\mathbb{P}\left(S^{2} \mathcal{B}^{\vee}\right)$ along $\mathbb{P}(V / \mathcal{A})$ and $\widetilde{Q}$ along $\mathbb{P}\left(S^{2} \mathcal{A}^{\vee}\right)$. They both give us the same variety, say $\bar{Q}$. In summary we have the following diagram from contractions in different orders:

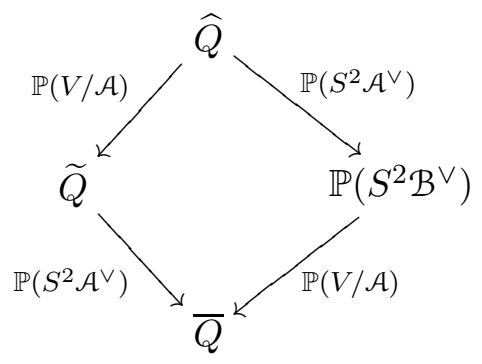

More generally, consider the vector bundle $\mathcal{W} \rightarrow J=\operatorname{Pic}^{0}(X)$ of rank $g$ given by $\mathcal{W}=R^{1} \pi_{J *} \mathcal{L}^{-2}(-x)$ where $\pi_{J}: J \times X \rightarrow J$ is the projection and $\mathcal{L} \rightarrow J \times X$ is a universal bundle. Let

$$
Q_{J}:=\mathbb{P H o m}\left(\operatorname{sl}(2)^{\vee}, \mathcal{W}\right) / / P G L(2)
$$

be the quotient of the projective bundle of homomorphisms of $\operatorname{sl}(2)^{\vee}$ to a fiber of $\mathcal{W} \rightarrow J$. Let $\widetilde{Q}_{J}$ be the blowup of $Q_{J}$ along the locus of rank 1 homomorphisms, i.e. the partial desingularization of $Q_{J}$. Then analogously as above we obtain the following.

Theorem 4.4. $\widetilde{Q}_{J}$ is isomorphic to the moduli space $\overline{\mathbf{M}}_{0,0}(\mathbb{P} \mathcal{W} / J, 2)$ of relative stable maps of degree 2 to $\mathbb{P W} \rightarrow J$.

The partial desingularization $\widetilde{Q}_{J}$ is singular along the proper transform

$$
\Delta_{J}:=\widetilde{\operatorname{PHom}}_{2}\left(\operatorname{sl}(2)^{\vee}, \mathcal{W}\right) / / P G L(2)
$$

of the locus of rank $\leq 2$ homomorphisms. The blowup $\widehat{Q}_{J}$ of $\widetilde{Q}_{J}$ along $\Delta_{J}$ is the variety of complete conics $C C\left(\mathcal{B}_{J}\right)$ where $\mathcal{B}_{J} \rightarrow G r(3, \mathcal{W})$ is the 
universal rank 3 bundle over the relative Grassmannian. The exceptional divisor is

$$
\widehat{\Delta}_{J}=\mathbb{P}\left(S^{2} \mathcal{A}_{J}^{\vee}\right) \times_{G r(2, \mathcal{W})} \mathbb{P}\left(\mathcal{W} / \mathcal{A}_{J}\right)
$$

where $\mathcal{A}_{J}$ is the universal rank 2 bundle over the Grassmannian $\operatorname{Gr}(2, \mathcal{W})$. Furthermore we have the following diagram by contractions:

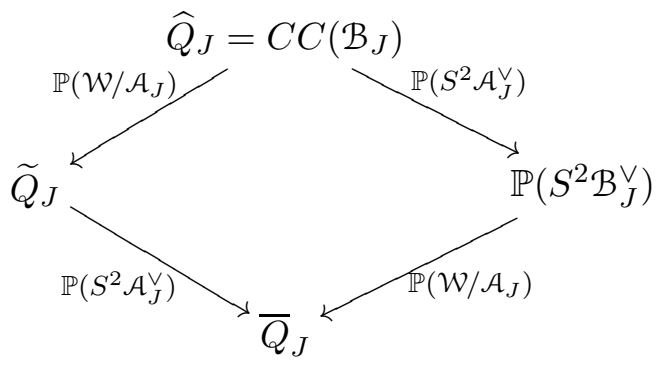

Theorem 4.4 and (4.8) hold similarly for $\mathcal{W}_{0} \rightarrow \tilde{X}$ instead of $\mathcal{W} \rightarrow J$ where $\mathcal{W}_{0}$ is the restriction of $\mathcal{W}$ via the embedding $\tilde{X} \hookrightarrow J$.

A degree 2 stable map $f: C \rightarrow \mathbb{P} H^{1}\left(\xi^{-2}(-x)\right)$ for $\xi \in J$ gives us a degree 2 stable map $f: C \rightarrow \mathcal{N}$ by composing with the embedding

$$
\mathbb{P} H^{1}\left(\xi^{-2}(-x)\right) \cong \mathbb{P} E x t^{1}\left(\xi, \xi^{-1}(-x)\right) \hookrightarrow \mathcal{N}
$$

by Proposition 2.6 . The bundle $\mathbb{P} \mathcal{W}$ parameterizes extension bundles of a degree 0 line bundle by a degree -1 line bundle and thus there is a natural morphism $\mathbb{P} \mathcal{W} \rightarrow \mathcal{N}$. By Theorem 4.4 and this morphism, we obtain the following.

Corollary 4.5. There is an injective morphism

$$
\lambda: \widetilde{Q}_{J} \rightarrow \overline{\mathbf{M}}_{0,0}(\mathcal{N}, 2)
$$

whose image parameterizes degree 2 stable maps of extension type.

Injectivity is a consequence of the following result of Narasimhan and Ramanan.

Proposition 4.6. 13, 6.19] Let $\xi_{1} \neq \xi_{2} \in J$. Then $\mathbb{P} H^{1}\left(\xi_{1}^{-2}(-x)\right)$ and $\mathbb{P} H^{1}\left(\xi_{2}^{-2}(-x)\right)$ intersect if and only if $\left(\xi_{1}, \xi_{2}\right) \in \mathcal{K}_{J}-\tilde{X}$. When they meet, they intersect at exactly one point transversely.

In $\S 6$, we will prove that the image of $\lambda$ is normal and hence $\lambda$ is an isomorphism by Zariski's main theorem.

\section{HeCke CORRESPONDENCE AND PARTIAL DESINGUlarization}

Let $\mathcal{M}_{X}=\mathcal{M}(2, X)$ (resp. $\mathcal{M}=\mathcal{M}\left(2, \mathcal{O}_{X}\right)$, resp. $\left.\mathcal{M}(2,0)\right)$ be the moduli space of rank 2 semistable bundles $E$ with $\operatorname{det} E \cong \mathcal{O}_{X}(y-x)$ for some 
$y \in X$ (resp. $\left.\operatorname{det} E \cong \mathcal{O}_{X}, \operatorname{resp} . \operatorname{deg} E=0\right)$. Then we have a Cartesian diagram

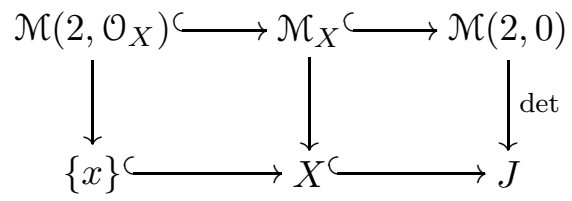

where the inclusion $X \hookrightarrow J$ is $y \mapsto \mathcal{O}_{X}(y-x)$.

The projectivization $q_{1}: \mathcal{H} \rightarrow \mathcal{N} \times X$ of a universal bundle over $\mathcal{N} \times X$ can be thought of as the moduli space of rank 2 bundles equipped with parabolic structure. Elementary modification using the parabolic structure gives us a family of rank 2 semistable bundles on $X$ with determinant $\mathcal{O}_{X}(y-x)$ for some $y \in X$, parameterized by $\mathcal{H}$ and hence we have a map $q_{0}: \mathcal{H} \rightarrow \mathcal{M}_{X}$. Therefore we have the Hecke correspondence

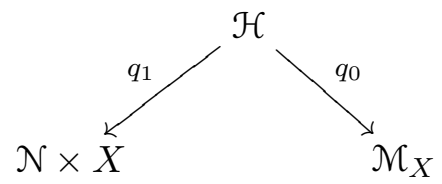

Although there is no universal bundle for $\mathcal{N}_{X}^{s}$, there is a projective universal bundle on $\mathcal{M}_{X}^{s} \times X$. The restriction $\mathcal{H}_{0}$ of this $\mathbb{P}^{1}$-bundle to $\mathcal{M}_{X}^{s}$ by the graph

$$
\alpha: \mathcal{M}_{X}^{s} \rightarrow \mathcal{M}_{X}^{s} \times X
$$

of the determinant map det $: \mathcal{M}_{X}^{s} \rightarrow X$ parameterizes a rank 2 stable bundle $E$ of determinant $\mathcal{O}_{X}(y-x)$ and a parabolic structure at $y$ for $y \in X$. It is elementary to set up moduli problems for $\mathcal{H}$ and $\mathcal{H}_{0}$ with parabolic bundles and see that $\mathcal{H}_{0}$ is the open subscheme $q_{0}^{-1}\left(\mathcal{M}_{X}^{s}\right)$ of $\mathcal{H}$. Therefore we have a family of stable maps

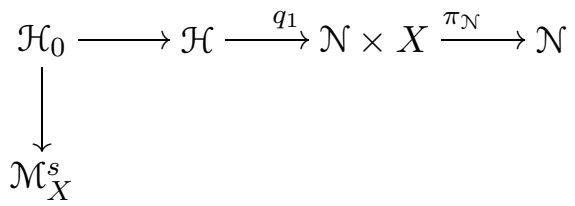

By definition, this is precisely the family of all Hecke curves in $\mathcal{N}$ and we obtain a morphism

$$
\psi^{\prime}: \mathcal{M}_{X}^{s} \rightarrow \overline{\mathbf{M}}_{0,0}(\mathcal{N}, 2)
$$

which is injective by Theorem 2.3 (3).

Lemma 5.1. $\psi^{\prime}$ is a birational morphism into an open subvariety of $\overline{\mathbf{M}}_{0,0}(\mathcal{N}, 2)$.

Proof. By Theorem 2.3 (2), the image of $\psi^{\prime}$ is contained in a nonsingular open subvariety of dimension $3 g-2=\operatorname{dim} \mathcal{N}_{X}^{s}$ (Remark 2.4). Hence $\psi^{\prime}$ is an injective morphism between nonsingular irreducible quasiprojective varieties of the same dimension. By considering the Jacobian determinant as in the proof of Theorem 3.7 $\psi^{\prime}$ is a birational morphism to an open subvariety of $\overline{\mathbf{M}}_{0,0}(\mathcal{N}, 2)$. 
We claim that $\psi^{\prime}$ extends to a morphism $\psi: \widetilde{\mathcal{M}}_{X} \rightarrow \overline{\mathbf{M}}_{0,0}(\mathcal{N}, 2)$ from the partial desingularization of $\mathcal{M}_{X}$.

Kirwan's partial desingularization has been defined and studied in 10. See also 8$] \S \S 3,5$. The singular locus of $\mathcal{M}_{X}$ is the locus of polystable bundles $E=\xi_{1} \oplus \xi_{2}$ for $\xi_{1}, \xi_{2} \in J=P i c^{0}(X)$, i.e.

$$
\mathcal{K}_{J}=\left\{\left(\xi_{1}, \xi_{2}\right) \in J \times J \mid \xi_{1} \xi_{2}=\mathcal{O}_{X}(y-x) \text { for some } y \in X\right\} / \mathbb{Z}_{2}
$$

where $\mathbb{Z}_{2}$ interchanges $\xi_{1}$ and $\xi_{2}$. If $\xi_{1} \neq \xi_{2}$, the singularity along $\mathcal{K}_{J}$ is by Luna's slice theorem

$$
\mathbb{C}^{g-1} \oplus \mathbb{C}^{g-1} / / \mathbb{C}^{*}
$$

where $\mathbb{C}^{*}$ acts with weights 1 and -1 on the summands respectively. When $\xi_{1}=\xi_{2}$, the singularity along $\tilde{X}=\left\{\xi \in J \mid \xi^{2}=\mathcal{O}_{X}(y-x)\right.$ for some $\left.y \in X\right\}$ is

$$
\operatorname{Hom}\left(\operatorname{sl}(2)^{\vee}, \mathcal{W}_{0}\right) / / P G L(2)
$$

where $\mathcal{W}_{0}=R^{1} \pi_{*} \mathcal{L}_{0}^{-2}(-x)$ is a vector bundle of rank $g$ over $\tilde{X}, \mathcal{L}_{0}$ being the restriction of a Poincaré bundle on $J \times X$ and $\pi: \tilde{X} \times X \rightarrow \tilde{X}$ the projection. To get the partial desingularization we blow up $\mathcal{M}_{X}$ first along the deepest stratum $\tilde{X}$ and then along the proper transform of the middle stratum $\mathcal{K}_{J}$. The exceptional divisor of the first blowup is

$$
\mathbb{P H o m}\left(\operatorname{sl}(2)^{\vee}, \mathcal{W}_{0}\right) / / P G L(2)=Q_{\tilde{X}}
$$

and its intersection with the proper transform of $\mathcal{K}_{J}$ is the locus of rank 1 homomorphisms

$$
\mathbb{P H o m}_{1}\left(\operatorname{sl}(2)^{\vee}, \mathcal{W}_{0}\right) / / P G L(2) .
$$

The proper transform of $Q_{\tilde{X}}$ after the second blowup is the partial desingularization

$$
\widetilde{Q}_{\tilde{X}}=b l_{\mathbb{P H o m}_{1}\left(s l(2)^{\vee}, \mathcal{W}_{0}\right)} \mathbb{P H o m}\left(\operatorname{sl}(2)^{\vee}, \mathcal{W}_{0}\right) / / P G L(2)
$$

and the singular locus of $\widetilde{\mathcal{M}}_{X}$ is precisely

$$
\Delta_{\tilde{X}}:=b l_{\mathbb{P H o m}_{1}\left(s l(2)^{\vee}, \mathcal{W}_{0}\right)} \mathbb{P H o m}_{2}\left(s l(2)^{\vee}, \mathcal{W}_{0}\right) / / P G L(2)
$$

and the singularities are $\mathbb{Z}_{2}$-quotients. From $\S 4$,

$$
\Delta_{\tilde{X}} \cong \mathbb{P}\left(S^{2} \mathcal{A}_{\tilde{X}}^{\vee}\right) \rightarrow G r\left(2, \mathcal{W}_{0}\right)
$$

where $\mathcal{A}_{\tilde{X}}$ is the universal rank 2 bundle over the Grassmannian bundle $\operatorname{Gr}\left(2, \mathcal{W}_{0}\right)$ over $\tilde{X}$. By blowing up $\tilde{\mathcal{M}}_{X}$ once again along $\Delta_{\tilde{X}}$ we get a (full) desingularization $\widehat{\mathcal{M}}_{X}$. As in Proposition 4.3, the proper transform $\widehat{Q}_{\tilde{X}}$ of the exceptional divisor $Q_{\tilde{X}}$ of the first blowup is

$$
C C\left(\mathcal{B}_{\tilde{X}}\right) \rightarrow \operatorname{Gr}\left(3, \mathcal{W}_{0}\right)
$$

and the exceptional divisor of the last blowup $\widehat{\mathcal{M}}_{X} \rightarrow \tilde{\mathcal{M}}_{X}$ is

$$
\widehat{\Delta}_{\tilde{X}}=\mathbb{P}\left(S^{2} \mathcal{A}_{\tilde{X}}^{\vee}\right) \times_{G r\left(2, \mathcal{W}_{0}\right)} \mathbb{P}\left(\mathcal{W}_{0} / \mathcal{A}_{\tilde{X}} \oplus \eta\right)
$$

for some line bundle $\eta$ on $\operatorname{Gr}\left(2, \mathcal{W}_{0}\right)$. Let $\widehat{\Sigma}$ be the proper transform of the exceptional divisor of the second blowup. For $B \in G r\left(3, \mathcal{W}_{0}\right)$, let $C C(B)$ 
denote the fiber of $C C\left(\mathcal{B}_{\tilde{X}}\right) \rightarrow G r\left(3, \mathcal{W}_{0}\right)$ over $B$. Then using the notation of $\S 4$, we have

$$
\begin{gathered}
{\left.[\widehat{\Sigma}]\right|_{C C(B)}=h_{3}} \\
{\left.\left[\widehat{\Delta}_{\tilde{X}}\right]\right|_{C C(B)}=-2 h_{1}+3 h_{2}-2 h_{3}} \\
\left.K_{\widehat{Q}_{\tilde{X}}}\right|_{C C(B)}=-(g-4) h_{1}-6 h_{2}+2 h_{3}
\end{gathered}
$$

by local computation. (See [8, §5].) Using (4.4), we obtain the following table of intersection numbers:

$$
\begin{array}{ccccc} 
& \widehat{Q}_{\tilde{X}} & \widehat{\Sigma} & \widehat{\Delta}_{\tilde{X}} & K_{\widehat{\mathcal{M}}_{X}} \\
\sigma_{1} & 0 & 2 & -1 & -2 \\
\sigma_{2} & 1 & 0 & -2 & -(g-3) \\
\sigma_{3} & -1 & -1 & 2 & -1
\end{array}
$$

In particular, the intersection numbers of the divisor class $\left[\widehat{\Delta}_{\tilde{X}}\right]$ with rational curves $C_{1}$ in the fibers of $\mathbb{P}\left(S^{2} \mathcal{A}_{\tilde{X}}^{\vee}\right)$ and $C_{2}$ in $\mathbb{P}\left(\mathcal{W}_{0} / \mathcal{A}_{\tilde{X}} \oplus \eta\right)$ are

$$
\left[\widehat{\Delta}_{\tilde{X}}\right] \cdot C_{1}=-1, \quad\left[\widehat{\Delta}_{\tilde{X}}\right] \cdot C_{2}=-2 .
$$

Hence the restriction of the normal bundle $\mathcal{O}_{\widehat{\mathcal{M}}_{X}}\left(\widehat{\Delta}_{\tilde{X}}\right)$ to the fibers $\mathbb{P}^{2} \times \mathbb{P}^{g-2}$ of $\widehat{\Delta}_{\tilde{X}} \rightarrow \operatorname{Gr}\left(2, \mathcal{W}_{0}\right)$ is $\mathcal{O}(-1,-2)$. The contraction of $\widehat{\mathcal{M}}_{X}$ along $\mathbb{P}\left(\mathcal{W}_{0} / \mathcal{A} \oplus \eta\right)$ gives us the orbifold $\tilde{\mathcal{M}}_{X}$ while the contraction of $\mathbb{P}\left(S^{2} \mathcal{A}_{\tilde{X}}^{\vee}\right)$ is a nonsingular variety $\mathcal{M}_{X}^{\#}$. We will prove in $\S 7$ that this nonsingular variety is one of the two irreducible components of the Hilbert scheme $\mathbf{H}$ of conics in $\mathcal{N}$. We can further contract $\mathbb{P}\left(\mathcal{W}_{0} / \mathcal{A}_{\tilde{X}} \oplus \eta\right)$ to obtain an orbifold $\mathcal{M}_{X}^{b}$ which turns out to be an irreducible component of the Chow scheme of conics in $\mathcal{N}$. In summary we have the following commutative diagram whose maps are all contractions (in different orders).

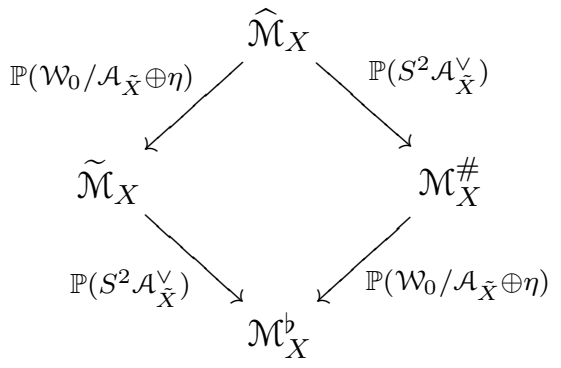

In [4] $\S \S 5,6$, we constructed a family of Hecke cycles parameterized by (a neighborhood of each point in) the desingularization $\widehat{\mathcal{M}}\left(2, \mathcal{O}_{X}\right)$ of $\mathcal{M}\left(2, \mathcal{O}_{X}\right)$ and during the proof a family of maps parameterized by the partial desingularization $\tilde{\mathcal{M}}\left(2, \mathcal{O}_{X}\right)$. It is straightforward to modify the constructions to obtain the following.

Theorem 5.2. The birational morphism $\psi^{\prime}: \mathcal{M}_{X}^{s} \rightarrow \overline{\mathbf{M}}_{0,0}(\mathcal{N}, 2)$ extends to an injective morphism

$$
\psi: \tilde{\mathcal{M}}_{X} \rightarrow \overline{\mathbf{M}}_{0,0}(\mathcal{N}, 2)
$$


The image of $\psi$ is an irreducible component parameterizing all stable maps of the following types:

(i) Hecke curves $f_{E, y}$ for $E \in \mathcal{M}_{X}^{s}$, $\operatorname{det} E \cong \mathcal{O}_{X}(y-x)$

(ii) degree 2 maps $f: C \rightarrow \mathbb{P} H^{1}\left(\xi^{-2}(-x)\right)$ for $\xi \in \tilde{X}$

(iii) unions of two intersecting lines $l_{1} \cup l_{2}$ for $l_{1} \subset \mathbb{P} H^{1}\left(\xi_{1}^{-2}(-x)\right), l_{2} \subset$ $\mathbb{P} H^{1}\left(\xi_{2}^{-2}(-x)\right),\left(\xi_{1}, \xi_{2}\right) \in \mathcal{K}_{J}-\tilde{X}$.

Proof. We leave the modification of [4] $\S \S 5,6$ to the reader. The local computation in the proofs of Lemma 5.2 and Lemma 6.1 shows that the stable maps parameterized by $\widetilde{\mathcal{M}}_{X}$ are of the above types.

In $\S 6$, we will prove that the image of $\psi$ is normal and thus $\psi$ is an isomorphism onto an irreducible component of $\overline{\mathbf{M}}_{0,0}(\mathcal{N}, 2)$.

\section{Moduli space of Stable maps of Degree 2 to $\mathcal{N}$}

In this section we prove Theorem 1.2. By Proposition 3.6, there are only 3 types of stable maps of degree 2 .

Lemma 6.1. A stable map of degree 2 to $\mathcal{N}$ is one of the following:

(i) Hecke curves $f_{E, y}$ for $E \in \mathcal{M}_{X}^{s}$, det $E \cong \mathcal{O}_{X}(y-x)$

(ii) degree 2 maps $f: C \rightarrow \mathbb{P} H^{1}\left(\xi^{-2}(-x)\right)$ for $\xi \in J=\operatorname{Pic}^{0}(X)$

(iii) union of two intersecting lines $l_{1} \cup l_{2}$ for $l_{1} \subset \mathbb{P} H^{1}\left(\xi_{1}^{-2}(-x)\right), l_{2} \subset$ $\mathbb{P} H^{1}\left(\xi_{2}^{-2}(-x)\right), \xi_{1} \neq \xi_{2} \in J$.

By Proposition 4.6] if $\mathbb{P} H^{1}\left(\xi_{1}^{-2}(-x)\right)$ and $\mathbb{P} H^{1}\left(\xi_{2}^{-2}(-x)\right)$ intersect, then $\left(\xi_{1}, \xi_{2}\right) \in \mathcal{K}_{J}-\tilde{X}$. Hence stable maps of type (iii) are all contained in the image of $\psi: \widetilde{\mathcal{M}}_{X} \rightarrow \overline{\mathbf{M}}_{0,0}(\mathcal{N}, 2)$ by Theorem 5.2 .

We proved in $\S 5$ that there is an injective morphism $\psi: \widetilde{\mathcal{M}}_{X} \rightarrow \overline{\mathbf{M}}_{0,0}(\mathcal{N}, 2)$ whose image is an irreducible component $\mathbf{M}_{H}$, which we call the Hecke component, parameterizing all stable maps of types (i), (iii) and stable maps of type (ii) with $\xi \in \tilde{X}$. We also proved in $\S 4$ that there is an injective morphism $\lambda: \widetilde{Q}_{J} \rightarrow \overline{\mathbf{M}}_{0,0}(\mathcal{N}, 2)$ whose image parameterizes stable maps of type (ii) with $\xi$ not necessarily in $\tilde{X}$. Since $\widetilde{Q}_{J}$ is irreducible projective and $\lambda$ is an injective map whose image contains the complement of the Hecke component, $\lambda$ is bijective onto an irreducible component $\mathbf{M}_{E}$, which we call the extension component. Obviously the intersection of the two components parameterizes degree 2 maps $f: C \rightarrow \mathbb{P} H^{1}\left(\xi^{-2}(-x)\right)$ for $\xi \in \tilde{X}$. Hence the intersection is the image of $\widetilde{Q}_{\tilde{X}}$ by both $\lambda$ and $\psi$.

The purpose of this section is to prove the following.

Proposition 6.2. The Hecke component $\mathbf{M}_{H}$ and the extension component $\mathbf{M}_{E}$ are normal varieties intersecting transversely along a subvariety isomorphic to $\widetilde{Q}_{\tilde{X}}$.

Proof of Theorem [1.2, As $\widetilde{\mathcal{M}}_{X}$ and $\widetilde{Q}_{J}$ are irreducible normal projective, $\lambda$ and $\psi$ are bijective morphisms onto normal varieties and thus birational 
(see the proof of Theorem 3.7]). By Zariski's main theorem, $\psi$ and $\lambda$ are isomorphisms.

The rest of this section is devoted to a proof of Proposition 6.2 We already know that $\overline{\mathbf{M}}_{0,0}(\mathcal{N}, 2)$ is normal at Hecke curves (Remark 2.4).

To prove normality at the stable maps of type (ii) and (iii) of Lemma 6.1 we need to know the restriction of the tangent bundle $T \mathcal{N}$ of $\mathcal{N}$ to rational curves in $\mathcal{N}$ of extension type.

Proposition 6.3. (1) Let $l$ be a line in $\mathbb{P} H^{1}\left(\xi^{-2}(-x)\right)$ for $\xi \in J$. Then we have

$$
\left.T \mathcal{N}\right|_{l} \cong \begin{cases}\mathcal{O}(2) \oplus \mathcal{O}(1)^{g-2} \oplus \mathcal{O}(-1)^{g-2} \oplus \mathcal{O}^{g} & \text { if } \xi \notin \tilde{X} \\ \mathcal{O}(2) \oplus \mathcal{O}(1)^{g-1} \oplus \mathcal{O}(-1)^{g-1} \oplus \mathcal{O}^{g-2} & \text { if } \xi \in \tilde{X}\end{cases}
$$

(2) If $f: \mathbb{P}^{1} \rightarrow \mathbb{P} H^{1}\left(\xi^{-2}(-x)\right)$ for $\xi \in J$ is of degree 2 , then

$$
f^{*} T \mathcal{N} \cong \begin{cases}\mathcal{O}(4) \oplus \mathcal{O}(2)^{g-2} \oplus \mathcal{O}(-2)^{g-2} \oplus \mathcal{O}^{g} & \text { if } \xi \notin \tilde{X} \\ \mathcal{O}(4) \oplus \mathcal{O}(2)^{g-1} \oplus \mathcal{O}(-2)^{g-1} \oplus \mathcal{O}^{g-2} & \text { if } \xi \in \tilde{X}\end{cases}
$$

Proof. For a line $l \subset \mathbb{P} H^{1}\left(\xi^{-2}(-x)\right) \cong \mathbb{P}^{g-1}, \xi \in J$, the tangent bundle of $\mathbb{P} H^{1}\left(\xi^{-2}(-x)\right)$ restricted to $l$ is

$$
\mathcal{O}(2) \oplus \mathcal{O}(1)^{g-2}
$$

The computation of the normal bundle of $\mathbb{P} H^{1}\left(\xi^{-2}(-x)\right)$ in $\mathcal{N}$ is similar to the proof of Lemma 2.7. We consider the universal extension bundle $\mathcal{U}$ over $\mathbb{P} H^{1}\left(\xi^{-2}(-x)\right) \times X$ :

$$
0 \rightarrow \pi_{X}^{*} \xi^{-1}(-x) \otimes \mathcal{O}_{\mathbb{P}}(1) \rightarrow \mathcal{U} \rightarrow \pi_{X}^{*} \xi \rightarrow 0
$$

By [13, 6.7], the tangent bundle $T \mathbb{P}$ of $\mathbb{P} H^{1}\left(\xi^{-2}(-x)\right)$ fits into an exact sequence

$$
0 \rightarrow T \mathbb{P} \rightarrow R^{1} \pi_{\mathbb{P}_{*}}\left(\mathcal{U} \otimes \pi_{X}^{*} \xi^{-1}\right) \rightarrow R^{1} \pi_{\mathbb{P}_{*}} \mathcal{O}=H^{1}\left(X, \mathcal{O}_{X}\right) \otimes \mathcal{O}_{\mathbb{P}} \rightarrow 0
$$

where $\pi_{\mathbb{P}}$ denotes the projection onto $\mathbb{P} H^{1}\left(\xi^{-2}(-x)\right)$. We also have an exact sequence

$$
0 \rightarrow \mathcal{U} \otimes \pi_{X}^{*} \xi^{-1} \rightarrow \operatorname{End}_{0} \mathcal{U} \rightarrow \pi_{X}^{*} \xi^{2}(x) \otimes \mathcal{O}_{\mathbb{P}}(-1) \rightarrow 0
$$

This gives us a diagram

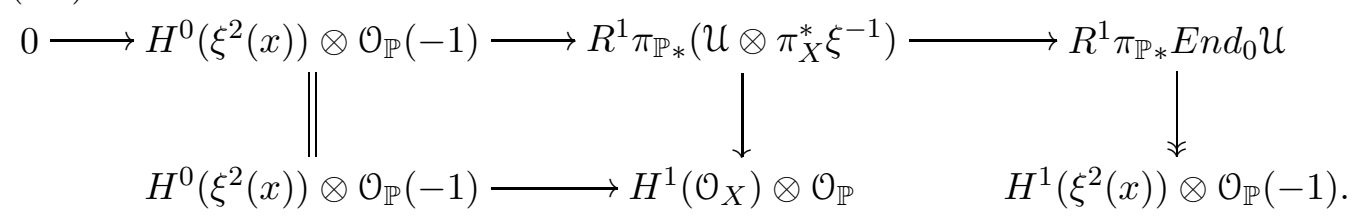

Suppose $\xi \notin \tilde{X}$. Then $H^{0}\left(\xi^{2}(x)\right)=0$ and $H^{1}\left(\xi^{2}(x)\right)=\mathbb{C}^{g-2}$. From (6.3), (6.4) and (6.6), we deduce that

$$
\left.T \mathcal{N}\right|_{l} \cong \mathcal{O}(2) \oplus \mathcal{O}(1)^{g-2} \oplus \mathcal{O}(-1)^{g-2} \oplus \mathcal{O}^{g}
$$

when $\xi \notin \tilde{X}$. 
Now suppose $\xi \in \tilde{X}$. Then $H^{0}\left(\xi^{2}(x)\right)=\mathbb{C}$ and $H^{1}\left(\xi^{2}(x)\right) \cong \mathbb{C}^{g-1}$. Since an extension bundle is uniquely determined by the epimorphism $E \rightarrow \xi$, we have

$$
\pi_{\mathbb{P}_{*}} \operatorname{Hom}\left(\pi_{X}^{*} \xi, \pi_{X}^{*} \xi\right) \cong \pi_{\mathbb{P}_{*}} \operatorname{Hom}\left(\mathcal{U}, \pi_{X}^{*} \xi\right)
$$

and thus the bottom horizontal in (6.6) is a monomorphism. Because

$$
H^{1}(\mathcal{O}) \otimes \mathcal{O}_{\mathbb{P}} / H^{0}\left(\xi^{2}(x)\right) \otimes \mathcal{O}_{\mathbb{P}}(-1) \cong \mathcal{O}(1) \oplus \mathcal{O}^{g-2}
$$

we deduce that

$$
\left.T \mathcal{N}\right|_{l} \cong \mathcal{O}(2) \oplus \mathcal{O}(1)^{g-1} \oplus \mathcal{O}(-1)^{g-1} \oplus \mathcal{O}^{g-2}
$$

when $\xi \in \tilde{X}$.

By the same computation for conics, we obtain (6.2).

We prove normality of $\overline{\mathbf{M}}_{0,0}(\mathcal{N}, 2)$ at the stable maps of type (iii) in Lemma 6.1. Let $f_{1}, f_{2}: \mathbb{P}^{1} \rightarrow \mathcal{N}$ be the lines $l_{1}, l_{2}$ with the intersection point $\theta$. Let $f: \mathbb{P}^{1} \cup \mathbb{P}^{1} \rightarrow \mathcal{N}$ be the morphism from the gluing of the points corresponding to $\theta$. From the exact sequence,

$$
\left.0 \rightarrow f^{*} T \mathcal{N} \rightarrow f_{1}^{*} T \mathcal{N} \oplus f_{2}^{*} T \mathcal{N} \rightarrow T \mathcal{N}\right|_{\theta} \rightarrow 0
$$

we get the exact sequence

$$
\begin{gathered}
0 \longrightarrow H^{0}\left(f^{*} T \mathcal{N}\right) \longrightarrow H^{0}\left(f_{1}^{*} T \mathcal{N}\right) \oplus H^{0}\left(f_{2}^{*} T \mathcal{N}\right) \longrightarrow H^{0}\left(\left.T \mathcal{N}\right|_{\theta}\right) \longrightarrow \\
\longrightarrow H^{1}\left(f^{*} T \mathcal{N}\right) \longrightarrow H^{1}\left(f_{1}^{*} T \mathcal{N}\right) \oplus H^{1}\left(f_{2}^{*} T \mathcal{N}\right) \longrightarrow 0
\end{gathered}
$$

By Proposition 6.3 (1), $H^{1}\left(f_{i}^{*} T \mathcal{N}\right)=0$ and $H^{0}\left(f_{i}^{*} T \mathcal{N}\right) \cong \mathbb{C}^{3 g-1}$. Hence normality at $[f]$ is a consequence of the following.

Lemma 6.4. $H^{0}\left(f_{1}^{*} T \mathcal{N}\right) \oplus H^{0}\left(f_{2}^{*} T \mathcal{N}\right) \rightarrow H^{0}\left(\left.T \mathcal{N}\right|_{\theta}\right)$ is surjective.

Proof. Because $\xi_{1} \neq \xi_{2}$ and $\xi_{1} \xi_{2}=\mathcal{O}_{X}(y-x)$, we have $\xi_{1}, \xi_{2} \notin \tilde{X}$. From (6.4) and (6.6), we have an isomorphism

$$
\left.T \mathcal{N}\right|_{\theta}=\left.T \mathbb{P} H^{1}\left(\xi_{1}^{-2}(-x)\right)\right|_{\theta} \oplus H^{1}(\mathcal{O}) \oplus H^{1}\left(\xi_{1}^{2}(x)\right)
$$

The three summands are respectively the subspaces of positive, zero and negative degrees for $\left.T \mathcal{N}\right|_{l_{1}}$. Note that $\left.T \mathbb{P} H^{1}\left(\xi_{1}^{-2}(-x)\right)\right|_{\theta}=H^{1}\left(\xi_{1}^{-2}(-x)\right) / \mathbb{C} e_{1}$ where $e_{1}$ is the extension class of

$$
0 \rightarrow \xi_{1}^{-1}(-x) \rightarrow E \rightarrow \xi_{1} \rightarrow 0
$$

at $\theta=[E]$. We also have an exact sequence

$$
0 \rightarrow \xi_{2}^{-1}(-x) \rightarrow E \rightarrow \xi_{2} \rightarrow 0
$$

and $\xi_{1}(-y) \cong \xi_{2}^{-1}(-x)$. Thus $\mathbb{C} e_{1}$ is the kernel of $H^{1}\left(\xi_{1}^{-2}(-x)\right)=\operatorname{Ext}^{1}\left(\xi_{1}, \xi_{1}^{-1}(-x)\right) \rightarrow \operatorname{Ext}^{1}\left(\xi_{2}^{-1}(-x), \xi_{1}^{-1}(-x)\right) \cong H^{1}\left(\xi_{1}^{-1} \xi_{2}\right)$. Therefore, $\left.T \mathbb{P} H^{1}\left(\xi_{1}^{-2}(-x)\right)\right|_{\theta} \cong H^{1}\left(\xi_{1}^{-1} \xi_{2}\right)=H^{1}\left(\xi_{2}^{2}(x-y)\right)$. From the exact sequence

$$
0 \rightarrow \xi_{2}^{2}(x-y) \rightarrow \xi_{2}^{2}(x) \rightarrow \mathcal{O}_{y} \rightarrow 0
$$


we get an exact sequence

$$
0 \rightarrow \mathbb{C} \rightarrow H^{1}\left(\xi_{2}^{2}(x-y)\right) \rightarrow H^{1}\left(\xi_{2}^{2}(x)\right) \rightarrow 0
$$

Hence (6.7) is now

$$
\left.T \mathcal{N}\right|_{\theta}=H^{1}\left(\xi_{2}^{2}(x)\right) \oplus \mathbb{C} \oplus H^{1}(\mathcal{O}) \oplus H^{1}\left(\xi_{1}^{2}(x)\right)
$$

The first three summands are the subspaces of nonnegative degrees for $T \mathcal{N}||_{l_{1}}$ while the last three summands are the subspaces of nonnegative degrees for $\left.T \mathcal{N}\right|_{l_{2}}$. This implies the desired surjectivity.

Now we consider the stable maps $f: C \rightarrow \mathcal{N}$ of type (ii) in Lemma 6.1 Suppose $\xi \notin \tilde{X}$. These stable maps are parameterized by the complement

$$
\overline{\mathbf{M}}_{0,0}(\mathcal{N}, 2)-\psi\left(\widetilde{\mathcal{M}}_{X}\right)
$$

which is an open set since $\widetilde{\mathcal{M}}_{X}$ is projective. By $(6.2)$,

$$
h^{1}\left(f^{*} T \mathcal{N}\right)=5+3(g-2)+g=4 g-1
$$

and hence the dimension of the deformation space at $[f] \in \overline{\mathbf{M}}_{0,0}(\mathcal{N}, 2)$ is constantly $4 g-4$ which is exactly the dimension of $\widetilde{Q}_{J}$. Hence the dimension of $\overline{\mathbf{M}}_{0,0}(\mathcal{N}, 2)-\psi\left(\tilde{\mathcal{M}}_{X}\right)$ is at most $4 g-4$. But we have a bijective morphism

$$
\lambda: \widetilde{Q}_{J}-\widetilde{Q}_{\tilde{X}} \rightarrow \overline{\mathbf{M}}_{0,0}(\mathcal{N}, 2)-\psi\left(\widetilde{\mathcal{M}}_{X}\right)
$$

By considering the Jacobian determinant, we deduce that $\overline{\mathbf{M}}_{0,0}(\mathcal{N}, 2)-$ $\psi\left(\widetilde{\mathcal{M}}_{X}\right)$ is irreducible of dimension exactly $4 g-4$ and hence it has at worst quotient singularities. In particular, $\overline{\mathbf{M}}_{0,0}(\mathcal{N}, 2)-\psi\left(\widetilde{\mathcal{M}}_{X}\right)$ is normal.

Finally let $f: C \rightarrow \mathcal{N}$ be a stable map of type (ii) with $\xi \in \tilde{X}$. Then $f$ is in the image of $\widetilde{Q}_{\tilde{X}}$ both by $\psi$ and by $\lambda$ if $\widetilde{Q}_{\tilde{X}}$ is considered as a divisor of $\widetilde{\mathcal{M}}_{X}$ by (5.1) and as a subvariety of $\widetilde{Q}_{J}$ by the embedding $\tilde{X} \hookrightarrow J$. Hence $f$ lies in the intersection of the two components $\mathbf{M}_{H}$ and $\mathbf{M}_{E}$. By (6.2), $h^{0}\left(f^{*} T \mathcal{N}\right)=4 g$. The proof of Proposition 6.2 is now complete if the deformation of $f$ in $\mathbf{M}_{H}$ is cut out by $(g-1)$ linear equations and the deformation of $f$ in $\mathbf{M}_{E}$ is cut out by 1 linear equation, transversal to the $(g-1)$-equations, on $H^{0}\left(f^{*} T \mathcal{N}\right)$.

Let us consider the deformation of $f$ in $\mathbf{M}_{E}$. By assumption $f$ factors through the projective bundle $\mathbb{P W}$ over $J$. The deformation of $f$ as a map to $\mathbb{P W}$ is from (6.5)

$$
H^{0}\left(R^{1} \pi_{\mathbb{P} *}\left(\mathcal{U} \otimes \pi_{X}^{*} \xi^{-1}\right)\right)
$$


using the notation of the proof of Proposition 6.3. By taking cohomology of (6.6) we obtain a diagram

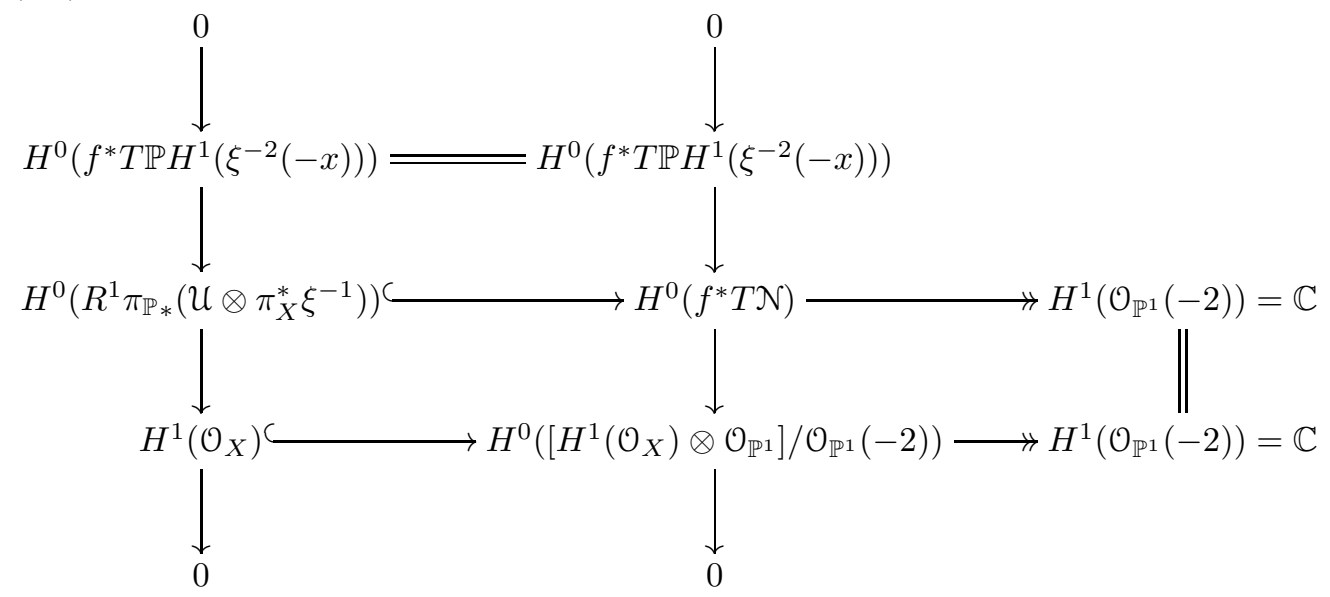

Therefore the deformation in $\mathbf{M}_{E}$ is cut out by one linear equation given by the surjective linear map $H^{0}\left(f^{*} T \mathcal{N}\right) \rightarrow H^{1}\left(\mathcal{O}_{\mathbb{P}^{1}}(-2)\right)=\mathbb{C}$ in (6.9). This implies the normality of the extension component $\mathbf{M}_{E}$.

Let us consider the deformation of $f$ in $\mathbf{M}_{H}$. Let $\tau$ be $\psi^{-1}(f) \in \widetilde{\mathcal{M}}_{X}$. The embedding $\tilde{X} \hookrightarrow J$ gives us a subspace $\mathbb{C} \hookrightarrow H^{1}\left(\mathcal{O}_{X}\right)$. The deformation space $V_{Q}$ of $f$ in the divisor $\widetilde{Q}_{\tilde{X}}$ of $\widetilde{\mathcal{M}}_{X}$ fits into a diagram

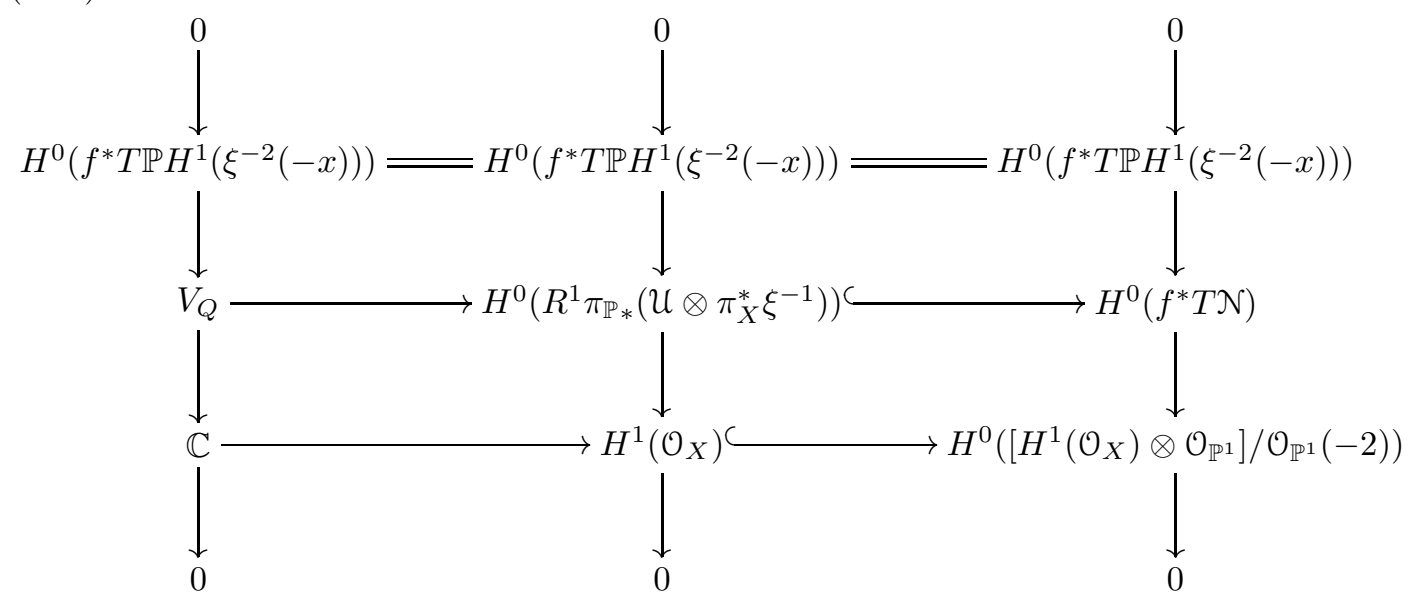

The dimension of $V_{Q}$ is at most $h^{0}\left(\mathcal{O}_{\mathbb{P}^{1}}(4) \oplus \mathcal{O}_{\mathbb{P}^{1}}(2)^{g-2}\right)+1=3 g$. To find the image of the deformation of $\tau$ in the normal direction of the divisor $\widetilde{Q}_{\tilde{X}}$ in $\widetilde{\mathcal{M}}_{X}$ we look at the local computation in [4, $\left.\S 6\right]$. By [4] (6.5), if we deform $f$ (or $\tau$ ) in the normal direction of $\widetilde{Q}_{\tilde{X}}$ in $\widetilde{\mathcal{M}}_{X}$, the diagonal part of the transition matrices gives us a normal vector field ( $z$ in the notation of [4]) of the divisor $\widetilde{Q}_{\tilde{X}}$ times the tangent vector field $\left(-a_{i j}-t c_{i j}\right.$ in [4]) of the conic $\left(b_{i j}-2 t a_{i j}-t^{2} c_{i j}\right.$ in [4]). In particular, it gives us $\mathcal{O}_{\mathbb{P}^{1}} \subset$ 
$\left[H^{1}\left(\mathcal{O}_{X}\right) \otimes \mathcal{O}_{\mathbb{P}^{1}}\right] / \mathcal{O}_{\mathbb{P}^{1}}(-2)$ since the pull-back of the normal bundle of $\widetilde{Q}_{\tilde{X}}$ by $f$ is $\mathcal{O}_{\mathbb{P}^{1}}(-2)$ by (5.3) . As the normal deformation is given by a nonconstant vector field $\left(-a_{i j}-t c_{i j}\right.$ in [4]), its image of the linear equation $H^{0}\left(f^{*} T \mathcal{N}\right) \rightarrow$ $\mathbb{C}$ for $\mathbf{M}_{E}$ is nonzero (see (6.9)). In summary, the deformation of $f$ in $\mathbf{M}_{H}$ is given by an extension of $\mathbb{C}$ by $V_{Q}$ which is transversal to $\mathbf{M}_{E}$ and is of codimension at least $g-1$. The proof is similar when $C$ is reducible. Therefore, $\mathbf{M}_{H}$ is normal everywhere and we finished a proof of Proposition 6.2 .

Remark 6.5. If one wants to find the moduli theoretic meaning of the partial desingularization of $\mathcal{M}=\mathcal{M}\left(2, \mathcal{O}_{X}\right)$ instead of $\mathcal{M}_{X}$, we just have to consider relative stable maps of degree 2

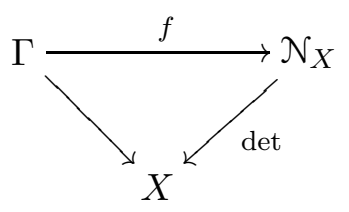

where $\Gamma$ is a flat family of semistable curves of genus 0 over $X$ and $\mathcal{N}_{X}$ is the moduli space of stable bundles of rank 2 with determinant $\mathcal{O}_{X}(-y)$ for some $y \in X$. We leave the details to the reader.

\section{Hilbert scheme And Chow scheme}

In this section we describe the Hilbert scheme $\mathbf{H}$ and the Chow scheme $\mathbf{C}$ of conics in $\mathcal{N}$, i.e. subschemes in $\mathcal{N}$ with Hilbert polynomial $2 m+1$. Let $\mathbf{M}=\overline{\mathbf{M}}_{0,0}(\mathcal{N}, 2)$ and $\widehat{\mathbf{M}}$ be the blowup of the disjoint union of the two components of $\mathbf{M}$ along the singular locus $\Delta_{J} \cup \Delta_{\tilde{X}}$.

We recall the following.

Lemma 7.1. 13 4.2,4.3] Let $C \subset \mathcal{N}$ be a conic. Then $C$ is isomorphic to a conic in $\mathbb{P}^{2}$ or a total thickening of a line $\mathbb{P}^{1}$ in $\mathbb{P}^{2}$.

Therefore $\mathbf{H}, \mathbf{C}$ and $\mathbf{M}$ are all isomorphic over the complement of the locus $\Delta_{J} \cup \Delta_{\tilde{X}}$ of double covers of a line in $\mathcal{N}$. Thus we have a diagram

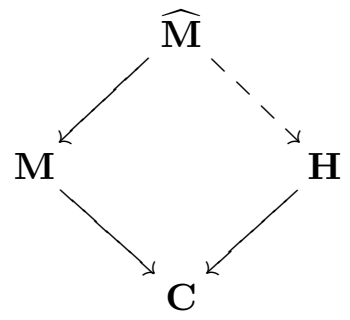

In 44 $\S \S 5,6]$, we constructed a family of Hecke cycles parameterized by (a neighborhood of each point of) the (full) desingularization of $\widehat{\mathcal{M}}$ and thus obtained a morphism from $\widehat{\mathcal{M}}$ to the Hilbert scheme of Hecke cycles. Further we proved the map is a blowup of the Hilbert scheme of Hecke cycles. It is straightforward to modify the constructions of [4] to prove the following. 
Theorem 7.2. There is a morphism $\widehat{\mathbf{M}} \rightarrow \mathbf{H}$ extending the birational map $\widehat{\mathbf{M}} \rightarrow \mathbf{H}$. Furthermore, the morphism is the contraction of $\mathbb{P}^{2}\left(S^{2} \mathcal{A}_{J}^{\vee}\right)$ via (4.7), (5.2).

Next let us consider the natural map from the Hilbert scheme $\mathbf{H}$ to the Chow scheme $\mathbf{C}$ (11]). This is not an isomorphism along the image of $\widehat{\Delta}_{J} \cup \widehat{\Delta}_{\tilde{X}}$. The points in $\operatorname{Gr}(2, \mathcal{W})$ determine a line $l$ in $\mathbb{P} \mathcal{W}$ and the fibers of $\mathbb{P}\left(\mathcal{W} / \mathcal{A}_{J}\right) \rightarrow \operatorname{Gr}(2, \mathcal{W})$ parameterize a thickening of $l$ by $\mathcal{O}(-1)$. Hence the morphism $\mathbf{H} \rightarrow \mathbf{C}$ factors through the contraction of $\mathbb{P}\left(\mathcal{W} / \mathcal{A}_{J}\right)$ for the extension component and of $\mathbb{P}\left(\mathcal{W} / \mathcal{A}_{\tilde{X}} \oplus \eta\right)$ for the Hecke component. The resulting map is one-to-one and the Chow scheme is seminormal. Therefore we deduce that the contraction of $\mathbf{H}$ is isomorphic to the Chow scheme $\mathbf{C}$. So we obtain the following.

Theorem 7.3. (1) The Hilbert scheme $\mathbf{H}$ of conics in $\mathcal{N}$ consists of two irreducible nonsingular components, intersecting transversely.

(2) $\mathbf{M}, \mathbf{H}, \mathbf{C}$ are related by (1.6) which is the diagram of contractions of $\widehat{\Delta}_{J} \cup \widehat{\Delta}_{\tilde{X}}$ in different orders.

\section{REFERENCES}

1. E. Brosius. Rank 2 vector bundles on a ruled surface. I. Math. Ann. 265 (1983), no. $2,155-168$.

2. S. Brivio and A. Verra. On the theta divisor of $\mathrm{SU}(2,1)$. Internat. J. Math. 10 (1999), no. 8, 925-942.

3. A.-M. Castravet. Rational families of vector bundles on curves. Internat. J. Math. 15 (2004), no. 1, 13-46.

4. I. Choe, J. Choy and Y.-H. Kiem. Cohomology of the moduli space of Hecke cycles. Topology 44 (2005), no. 3, 585-608.

5. D. Huybrechts and M. Lehn. The geometry of moduli spaces of sheaves. A Publication of the Max-Planck-Institut für Mathematik, Bonn (1997)

6. L. Jeffrey, Y.-H. Kiem, F. Kirwan and J. Woolf. Cohomology pairings on singular quotients in geometric invariant theory. Transform. Groups 8 (2003), no. 3, 217-259.

7. L.Jeffrey, Y.-H. Kiem, F. Kirwan and J. Woolf. Intersection pairings on singular moduli spaces of bundles over a Riemann surface. Preprint: math.AG/0505362

8. Y.-H. Kiem and J. Li. Desingularizations of the moduli space of rank 2 bundles over a curve. Math. Ann. 330 (2004), 491-518.

9. F. Kirwan. Partial desingularisations of quotients of nonsingular varieties and their Betti numbers. Ann. of Math. 122 (1985), 41-85.

10. F. Kirwan. On the homology of compactifications of moduli spaces of vector bundles over a Riemann surface. Proc. London Math. Soc. (3) 53 (1986), no. 2, 237-266.

11. J. Kollar. Rational curves on algebraic varieties. Springer-Verlag, Berlin, 1996.

12. V. Munoz. Quantum cohomology of the moduli space of stable bundles over a Riemann surface. Duke Math. J. 98 (1999), no. 3, 525-540.

13. M.S. Narasimhan and S. Ramnana. Geometry of Hecke cycles, I. C. P. Ramanujam - a tribute, pp. 291-345, Tata Inst. Fund. Res. Studies in Math., 8, Springer, Berlin-New York, 1978.

14. X. Sun. Minimal rational curves on moduli spaces of stable bundles. Math. Ann. 331 (2005), no. 4, 925-937. 
Department of Mathematics and Research Institute of Mathematics, Seoul National University, Seoul 151-747, Korea

Department of Mathematics, Stanford University, Stanford, USA

E-mail address: kiem@math.snu.ac.kr 